\title{
Mapping Lamb, Stark, and Purcell Effects at a Chromophore-Picocavity Junction with Hyper-Resolved Fluorescence Microscopy
}

\author{
Anna Rosławska $\odot,{ }^{1, *, \dagger}$ Tomáš Neuman $\odot,{ }^{1,2, *,+}$ Benjamin Doppagne, ${ }^{1}$ Andrei G. Borisov $\odot,{ }^{3}$ Michelangelo Romeo $\odot,{ }^{1}$ \\ Fabrice Scheurer, ${ }^{1}$ Javier Aizpurua ${ }^{2},{ }^{2}$ and Guillaume Schull ${ }^{1, \$}$ \\ ${ }^{1}$ Université de Strasbourg, CNRS, IPCMS, UMR 7504, F-67000 Strasbourg, France \\ ${ }^{2}$ Center for Materials Physics (CSIC-UPV/EHU) and DIPC, Paseo Manuel de Lardizabal 5, \\ Donostia-San Sebastián 20018, Spain \\ ${ }^{3}$ Institut des Sciences Moléculaires d'Orsay (ISMO), UMR 8214, CNRS, \\ Université Paris-Saclay, 91405 Orsay Cedex, France
}

(Received 30 April 2021; revised 29 September 2021; accepted 3 November 2021; published 19 January 2022)

\begin{abstract}
The interactions of the excited states of a single chromophore with static and dynamic electric fields spatially varying at the atomic scale are investigated in a joint experimental and theoretical effort. In this configuration, the spatial extension of the fields confined at the apex of a scanning tunneling microscope tip is smaller than that of the molecular exciton, a property used to generate fluorescence maps of the chromophore with intramolecular resolution. Theoretical simulations of the electrostatic and electrodynamic interactions occurring at the picocavity junction formed by the chromophore, the tip, and the substrate reveal the key role played by subtle variations of Purcell, Lamb, and Stark effects. They also demonstrate that hyper-resolved fluorescence maps of the line shift and linewidth of the excitonic emission can be understood as images of the static charge redistribution upon electronic excitation of the molecule and as the distribution of the dynamical charge oscillation associated with the molecular exciton, respectively.
\end{abstract}

DOI: $10.1103 /$ PhysRevX.12.011012

\section{INTRODUCTION}

Seminal experiments demonstrating the possibility to study optical properties of individual molecular chromophores $[1,2]$ gave rise to the development of superresolution fluorescence techniques-revolutionizing the field of optical microscopy $[3,4]$ —and inspired the engineering of molecule-based quantum-optical devices [5,6]. At the core of many of these applications stands the possibility to control the optical and electronic properties of molecules via static and dynamical electromagnetic interactions. In the weak-coupling limit, the interaction between the chromophore and its electromagnetic environment fastens the radiative decay of the emitter (Purcell effect $[7,8]$ ) and shifts its emission energy to the red (Lamb shift [9-12]). Electrostatic fields, on the other hand, cause Stark shifts $[13,14]$ of the emission lines or electronic states [15-17]. Nowadays, these effects are well understood in

\footnotetext{
*These authors contributed equally to this work.

†anna.roslawska@ipcms.unistra.fr

*tomas.neuman@ipcms.unistra.fr

§uillaume.schull@ipcms.unistra.fr
}

Published by the American Physical Society under the terms of the Creative Commons Attribution 4.0 International license. Further distribution of this work must maintain attribution to the author(s) and the published article's title, journal citation, and DOI.
Subject Areas: Condensed Matter Physics, Plasmonics

settings where the chromophore can be treated as a pointlike emitter exposed to homogeneous external electromagnetic fields.

The opposite limit, where the physical dimensions of the field confinement are small or comparable with the size of the molecular emitter, remains largely unexplored. Here, we address this extreme limit of the light-matter interaction by analyzing the spectra of light emission from a single electrically driven molecule in an atomically sharp tunneling junction of a scanning tunneling microscope (STM). We show how the Purcell effect, the Lamb shift, and the Stark shift can be exploited to elucidate the electronic and optical properties of individual molecules and their interaction with a plasmonic cavity, enabling ultimate spatial resolution by combining scanning tunneling microscopy and optical spectroscopy.

With respect to optical imaging of single molecules, scanning near-field optical microscopy [18-25] has proven to be a valuable tool thanks to its ability to confine electromagnetic fields at the extremity of the microscope tip. This path has been explored to its limits in tip-enhanced Raman spectroscopy (TERS) [26,27] and tip-enhanced photoluminescence (TEPL) [28] to investigate vibronic and fluorescence signals with submolecular precision. Scanning tunneling microscope-induced luminescence (STML), which uses tunneling electrons as an excitation source rather than photons, provided similar, if not better, spatial resolution over fluorescence signals of 
chromophores [29-45]. In typical STML experiments, hyper-resolved fluorescence microscopy (HRFM) maps are recorded by collecting the intensity of the photon emission as a function of atomic-scale variations of the STM tip position with respect to the molecule. These HRFM maps, however, are challenging to interpret because the excitation and emission mechanisms driven by electronic and optical processes are simultaneously at play.

In contrast to these previous STML experiments, we show that HRFM maps of molecular electroluminescence lines embed information on the molecule, as well as on its electromagnetic and electrostatic environment, encoded in the atomically resolved linewidths and line shifts. Based on a comparison between experimental data and theoretical calculations obtained for a free-base phthalocyanine $\left(\mathrm{H}_{2} \mathrm{Pc}\right)$ chromophore, we demonstrate that linewidth maps directly reflect the large Purcell effect generated by the coupling between the chromophore and gap plasmons at the tunneling junction, providing access to variations of the molecular excited-state lifetime with tip position. Therefore, this signal solely reflects the optical characteristics of the chromophore and can be used to generate subnanometric fluorescence maps of the molecular transition charge density, as we detail below. On the other hand, the energy of the chromophore fluorescence line depends on a subtle interplay between photonic Lamb $[28,38,46]$ and Stark shifts $[47,48]$ induced by the presence of the tip. Whereas the former provides intimate details of the exciton-plasmon coupling, the latter reveals information about the transfer of charges associated with the chromophore electronic transition. In addition, our theoretical calculations highlight the decisive role played by the extreme spatial variation of the electromagnetic fields at the tip apex - a geometry dubbed as a plasmonic picocavity $[27,28,49-56]$ - that is responsible for the ultimate spatial resolution in HRFM maps. The main advantage of our approach based on the mapping of the Purcell, Lamb, and Stark effects is that it is not hampered by the excitation probability of the chromophore, and it solely reflects the optical (linewidth maps) and electronic (line-shift map) characteristics of the exciton. Overall, this approach simultaneously provides access to the electronic and optical properties of chromophores with a close-to-atomic resolution, and decisive atomic-scale information on fundamental plasmon-exciton interactions in the limit where the spatial scales of the confined fields are comparable to that of the quantum emitter.

\section{PURCELL, LAMB, AND STARK EFFECTS IN STML}

The experimental approach [Fig. 1(a)] consists in using the tunneling electrons injected by the STM tip to excite the fluorescence of individual $\mathrm{H}_{2} \mathrm{Pc}$ molecules separated from a $\mathrm{Ag}(111)$ surface by three insulating monolayers of $\mathrm{NaCl}$. In such a system, the insulating layer is sufficiently thick to prevent the quenching of the fluorescence due to the direct (a)

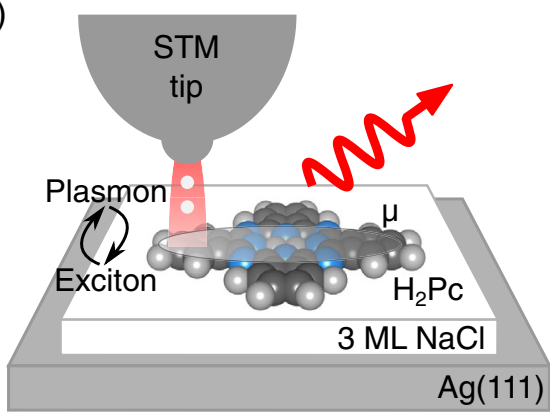

(b)

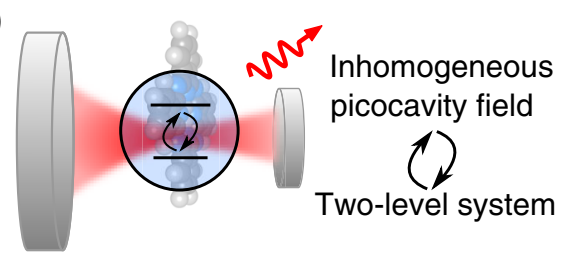

(c)

(d)
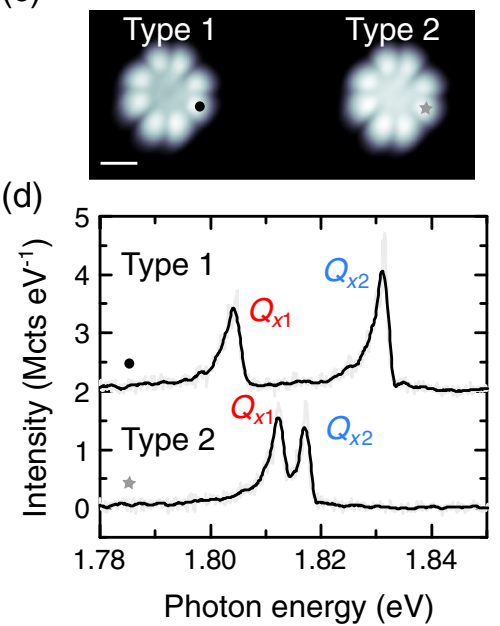

(e)

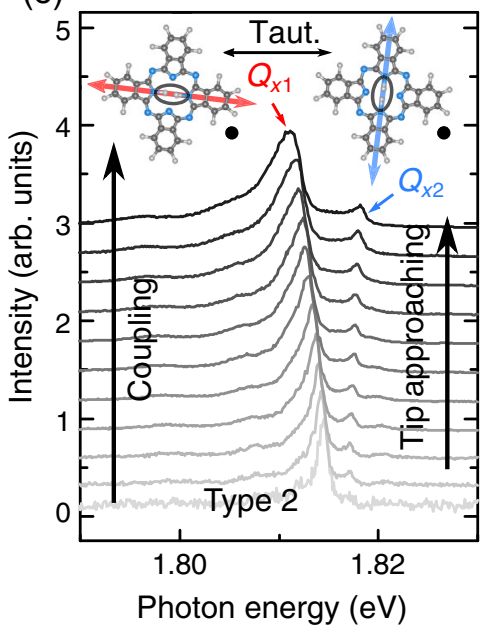

FIG. 1. (a) Sketch of the experiment where the plasmonic silver tip of a STM is used to excite the fluorescence of a single $\mathrm{H}_{2} \mathrm{Pc}$ molecule deposited on a NaCl-covered $\mathrm{Ag}(111)$ sample. The orientation of the molecular dipole $(\boldsymbol{\mu})$ is schematically marked by a gray ellipse. (b) Sketch of the system where an extended two-level system (molecule) interacts with the confined electromagnetic field of the plasmonic picocavity. (c) STM image $(V=-2.5 \mathrm{~V}, I=10 \mathrm{pA}$, scale bar: $1 \mathrm{~nm})$. (d) STML spectra $(V=-2.5 \mathrm{~V}, I=200 \mathrm{pA}$, $t=120 \mathrm{~s}$ ) of a type- 1 and a type- $2 \mathrm{H}_{2} \mathrm{Pc}$ (see main text for detail) acquired at positions marked by a black dot and gray star in panel (c). (e) Successive STML spectra acquired during the vertical approach of the STM tip to a $\mathrm{H}_{2} \mathrm{Pc}$ molecule of type 2. Note that $V=-2.5 \mathrm{~V}$, that $I$ ranges from $2 \mathrm{pA}$ (bottom spectrum) to $500 \mathrm{pA}$ (top spectrum) whereas $t$ ranges from $360 \mathrm{~s}$ to $30 \mathrm{~s}$. Inset: ball-and-stick models of two $\mathrm{H}_{2} \mathrm{Pc}$ tautomers with the $Q_{x 1}$ and $Q_{x 2}$ dipoles marked by red and blue arrows, respectively. The hydrogen atoms are marked by ellipses. The black dots indicate the tip location. 
contact between chromophores and metallic surfaces. The sketch also represents the interaction between plasmons confined at the extremity of the STM tip and the transition dipole moment $\boldsymbol{\mu}$ of the $\mathrm{H}_{2} \mathrm{Pc}$ molecule. In this configuration, the electromagnetic field confined at the plasmonic picocavity created by the atomically sharp tip couples with a molecular two-level system of larger spatial dimensions [Fig. 1(b)], in strong contrast to the homogeneous field distribution generally obtained in more standard electromagnetic cavities.

The topographic STM image in Fig. 1(c) shows two $\mathrm{H}_{2} \mathrm{Pc}$ molecules adsorbed on a $\mathrm{NaCl}$ island. This image reveals a slightly dimmer contrast in the center of the left molecule (labeled type 1), as compared to the right molecule (labeled type 2). This behavior has been attributed to slightly different geometries of the two molecules adsorbed on top of the $\mathrm{NaCl}$ ionic crystal [42]. The difference in adsorption geometries also affects the fluorescence properties of the molecules, as can be seen in the STML spectra provided in Fig. 1(d). Here, the molecules were driven to their excited states by tunneling electrons at the bias voltage $V=-2.5 \mathrm{~V}$ applied to the sample, and the tip-assisted light emission of these molecular excitons was detected in the far field. These high-resolution spectra show the main fluorescence transition $Q_{x}$ of $\mathrm{H}_{2} \mathrm{Pc}$, which is associated with a dipole moment oriented along the two hydrogens of the central core of the molecule. In Fig. 1(d), this transition appears as a doublet of lines labeled $Q_{x 1}$ and $Q_{x 2}$, whose energy separation is different for type-1 and type-2 molecules. This duplication of the $Q_{x}$ line has been attributed to the fast tautomerization of the central hydrogens within $\mathrm{H}_{2} \mathrm{Pc}$ during the acquisition of a STML spectrum [Fig. 1(e)] [42]. The positions of the hydrogen atoms are marked by ellipses in the inset of Fig. 1(e). When recording time-integrated spectra, one simultaneously records the fluorescence of both tautomers. The STML spectra of type-1 molecules feature a large difference between the $Q_{x 1}$ and $Q_{x 2}$ excitonic lines, which has been attributed to substantially different adsorption configurations of the two tautomers on the $\mathrm{NaCl}$ surface [42]. Type- 1 molecules can therefore be used to unambiguously separate the spectral contributions of $Q_{x 1}$ and $Q_{x 2}$, a property that we exploit in Sec. III. As we detail in Sec. IV, the small energy difference in the case of type- 2 molecules finds its origin in the coupling of the exciton with the electromagnetic fields induced in the presence of the STM tip. The first evidence of the influence of this coupling is provided in Fig. 1(e), where the spectral characteristics of a type- 2 molecule are monitored as a function of the vertical tip-molecule distance. By laterally positioning the tip as marked in Fig. 1(e), we ensure that $Q_{x 1}$ and $Q_{x 2}$ experience a nonequivalent interaction with the electromagnetic environment induced by the tip. Similarly to what was reported in Ref. [38], this distance-dependent spectrum reveals a shift of the $Q_{x 1}$ line towards lower energies when the tip-molecule distance is reduced [Fig. 1(e)]. In contrast, we observe a shift of the $Q_{x 2}$ line towards higher energies. In both cases, these energy shifts are accompanied by a systematic enlargement of the $Q_{x 1}$ and $Q_{x 2}$ linewidths.

To interpret these line shifts and line broadenings, we developed a theoretical approach accounting for the electromagnetic interactions occurring between the chromophore and the plasmonic picocavity [Fig. 2(a)]. Additional details are provided in Appendix A. First, we consider the exicton transition charge density $\rho_{\text {eg }}$ [Fig. 2(b)] for the optical transition at frequency $\omega_{\text {eg }}$ between the ground (g) and excited (e) electronic states of the molecule, calculated using timedependent density-functional theory (TD-DFT) [57]. The transition charge density can be viewed as an oscillating electron charge acting as a source stimulating the plasmonic response of the cavity, which here takes the form of the induced plasmonic potential $\phi_{\text {ind }}$ [Fig. 2(b)]. This potential then acts back on the exciton, causing a line shift $\hbar \delta \omega_{\text {eg }}=\operatorname{Re}\left\{\int \rho_{\text {eg }}(\mathbf{r}) \phi_{\text {ind }}(\mathbf{r}) d^{3} \mathbf{r}\right\}$ (Lamb shift), and broadens the excitonic emission line by $\hbar \gamma_{\mathrm{eg}}=$ $-2 \operatorname{Im}\left\{\int \rho_{\text {eg }}(\mathbf{r}) \phi_{\text {ind }}(\mathbf{r}) d^{3} \mathbf{r}\right\}$ (Purcell effect). Here, $\hbar$ is the reduced Planck constant. We additionally consider that the molecule is exposed to a strong inhomogeneous electrostatic potential $\phi_{\text {ext }}$ resulting from the bias applied between the tip and the substrate [Fig. 2(c)]. This potential modifies the energies of the electronic ground and excited states via the dc Stark effect, resulting in another shift of the excitonic energy $\delta E_{\mathrm{St}}=\int \Delta \rho(\mathbf{r}) \phi_{\mathrm{ext}}(\mathbf{r}) d^{3} \mathbf{r}$, which is merely the energy difference resulting from the redistribution of charge (charge transfer) associated to the electronic excitation in the molecule. Here, $\Delta \rho=\rho_{\mathrm{e}}-\rho_{\mathrm{g}}$ is the difference between the excited-state $\left(\rho_{\mathrm{e}}\right)$ and groundstate $\left(\rho_{\mathrm{g}}\right)$ charge densities [Fig. 2(c)].

Armed with this theoretical framework, we compare the line shifts and linewidths recorded experimentally (full squares) with the theoretical prediction (empty circles) and plot them as a function of the relative tip-sample distance in Fig. 2(d) and 2(f). Details about the tip geometry are provided in Fig. 6 of Appendix A. These approach curves are plotted for two excitons $Q_{x 1}$ and $Q_{x 2}$, whose orientations with respect to the tip are nonequivalent. We obtain the experimental data by fitting the spectra shown in Fig. 1(e) using the procedure described in Appendix B.

As the experimentally available tip-sample distance and line shifts are relative, in the theoretically calculated data, we choose the origin of the tip-substrate distance (i.e., the gap size) guided by the measured values, and we infer from the experimental data that the frequency of the exciton of the molecule on the substrate unperturbed by the tip is about $1.815 \mathrm{eV}$, as detailed in Appendix C. We observe two qualitatively different trends for the total line shift shown in Fig. 2(d) for $Q_{x 1}$ (red markers) and $Q_{x 2}$ (blue 
(a)

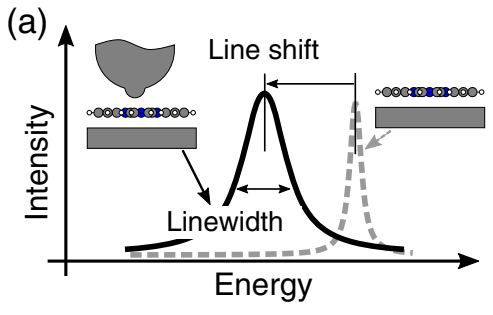

(d)

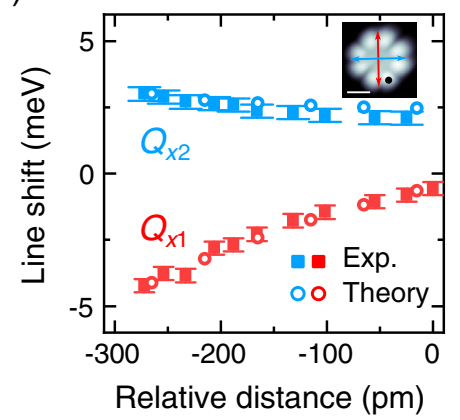

(b)

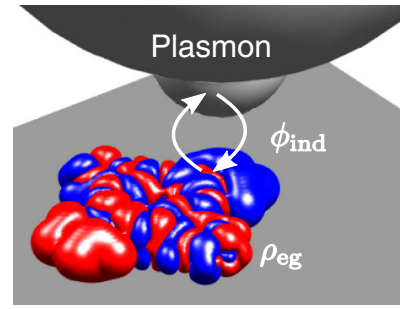

(e)

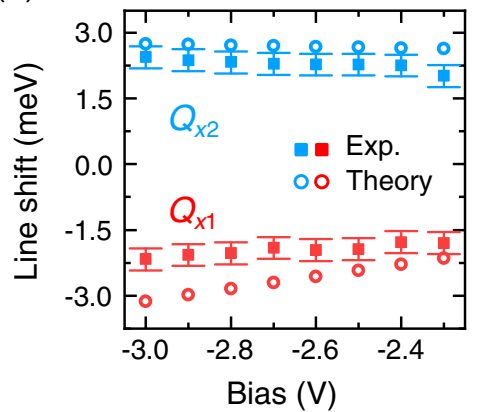

(c)

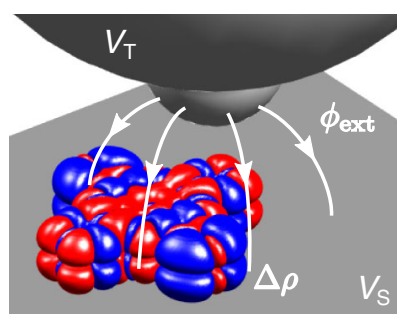

(f)

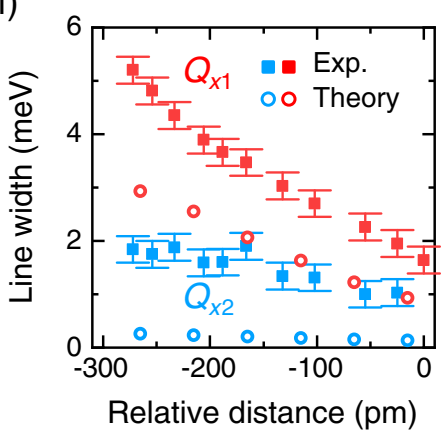

FIG. 2. Probing and calculating the dc Stark effect, Lamb shift, and Purcell effect in STML. (a) Schematic representation of the overall shift and broadening of the excitonic photon emission spectral line recorded in STML as the tip approaches the sample molecule. The two schemes show the systems used to model the STM junction; the molecule is separated from the substrate by a vacuum gap of thickness $d=0.5 \mathrm{~nm}$. (b) The excitonic transition charge density $\rho_{\mathrm{eg}}(\mathbf{r})$ induces a plasmonic response in the tip and the substrate, which is represented by the induced plasmonic potential $\phi_{\text {ind }}$. (c) Difference between the electron densities of the molecular electronic excited state $\left(Q_{x}\right)$ and the ground state. The charge density difference $\Delta \rho(\mathbf{r})$ is exposed to the external potential $\phi_{\text {ext }}(\mathbf{r})$ generated by the voltage $V=V_{\mathrm{S}}-V_{\mathrm{T}}$ applied to the substrate and the tip of the microscope. Red and blue indicate negative and positive values, respectively. (d) Line shift as a function of the relative tip-sample distance $(V=-2.5 \mathrm{~V})$. Inset: STM image $V=-2.5 \mathrm{~V} ; I=10 \mathrm{pA}$; scale bar, $1 \mathrm{~nm}$. The black dot marks the position of the measurements in panels (d)-(f). The color arrows indicate the two dipoles. (e) Line shifts as a function of bias. The tip-sample distance is fixed during the measurement. The line shifts in panels (d) and (e) were calculated by taking $1.815 \mathrm{eV}$ as a reference value. (f) Linewidths as a function of the relative tip-sample distance $(V=-2.5 \mathrm{~V})$. The overall shifts (Lamb shift combined with the Stark shift) (d, e) and widths (f) of the excitonic emission lines experimentally recorded (full squares) are compared with the theoretical calculations (empty circles) obtained using the approach presented in panels (a)-(c). The data in panels (d) and (f) are extracted from the measurement shown in Fig. 1(e).

markers) excitons as a function of the relative tip-sample distance. The excitonic line of $Q_{x 1}$ is redshifting as the tip approaches, while the opposite trend is observed for $Q_{x 2}$. As we detail in Appendix C, in our setup, the Lamb effect always redshifts the exciton frequency irrespective of the tip position. The Stark effect can either redshift or blueshift the exciton frequency, depending on the lateral tip position. In Appendix C, we show that the Stark and Lamb effects jointly lower the frequency of $Q_{x 1}$ for the tip position marked by a black dot in the inset of Fig. 2(d). For the same tip position, the Stark effect blueshifts the $Q_{x 2}$ exciton and overrides the Lamb effect. The role played by the Stark effect is confirmed experimentally [see Fig. 2(e) and Appendix D] by monitoring the $Q_{x 1}$ and $Q_{x 2}$ line positions as a function of voltage while keeping the tip-molecule distance fixed. In this configuration, the plasmon-exciton coupling is constant. The line shifts in that situation are therefore exclusively related to the increasing electrical field and thus directly measure the Stark shift. This effect is accounted for by our model [empty circles in Fig. 2(e)].

In Fig. 2(f), experiment and theory also report an increasing linewidth with decreasing tip-sample distance for $Q_{x 1}$ and, to a smaller extent, $Q_{x 2}$. In the theory, this reflects the increased decay rate of the exciton into plasmons by the Purcell effect. The experiments reveal a similar behavior, with an offset of about $1 \mathrm{meV}$ suggesting an additional decoherence channel not accounted for in the simulations (e.g., interaction of the excitons with substrate phonons or low-energy molecular vibrations). These effects are further discussed in Appendix B. As the experimental tendencies are well reproduced by our theoretical predictions, we can tentatively assign the component of the line broadening that depends on the tip position to the plasmonic Purcell effect. Overall, this indicates an excited-state lifetime of the order of $1 \mathrm{ps}$ - about 3 orders of magnitude shorter than what is expected for the free molecule [58,59] -and highlights the strong amplification of the chromophore emission provided by the plasmonic picocavity. The plasmon-induced line broadening recorded for $Q_{x 1}$ is considerably larger than for $Q_{x 2}$ as the relative orientations of the excitonic dipolar moments with respect to the tip differ for the two excitons, resulting in larger plasmonexciton coupling strengths for $Q_{x 1}$ than for $Q_{x 2}$. Importantly, within the first order approximation, the 
plasmon-induced broadening of the excitonic line is expected to be free of the influence of the electrostatic field (which only results in the energy shift of the line). The modification of the linewidth therefore provides a direct measure of the plasmon-exciton coupling.

\section{MAPPING THE PURCELL, LAMB, AND STARK EFFECTS}

The shifts and broadening of the fluorescence lines induced by the Stark, Lamb, and Purcell effects suggest a modality of STML based on the mapping of these signals.
This technique could be used to acquire hyper-resolved information on the molecular excitons and their coupling to the picocavity plasmons.

To demonstrate this capability, we have recorded fluorescence spectra at each pixel of a constant-height STM current image [Fig. 3(a)] of a type- $1 \mathrm{H}_{2} \mathrm{Pc}$. Since only the effect of the lateral position of the tip is of interest here, the map was recorded in constant-height mode to avoid any changes of the tip-molecule distance that would affect the measurement of the line shift and linewidth. For reference, we also show the molecular geometry in Fig. 3(i). (a) Constant-height STM image

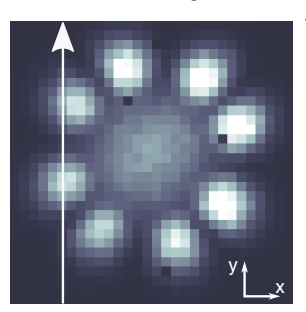

Photon intensity
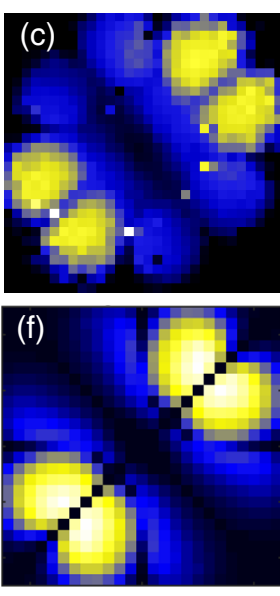

$\mathrm{H}_{2} \mathrm{Pc}$ geometry

(i)

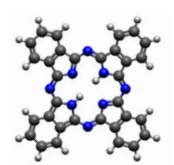

(b)
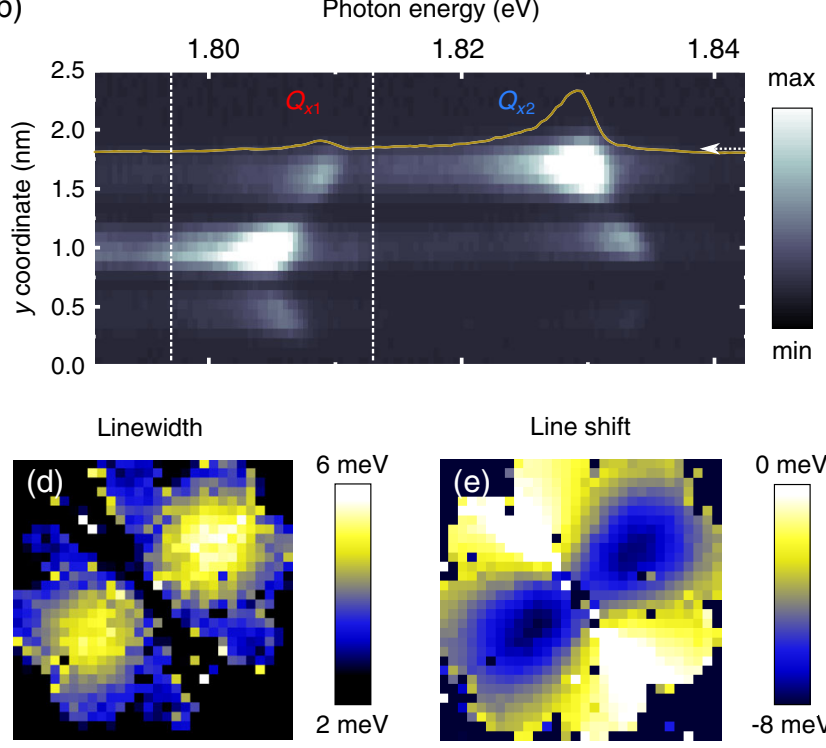

$0 \mathrm{meV}$
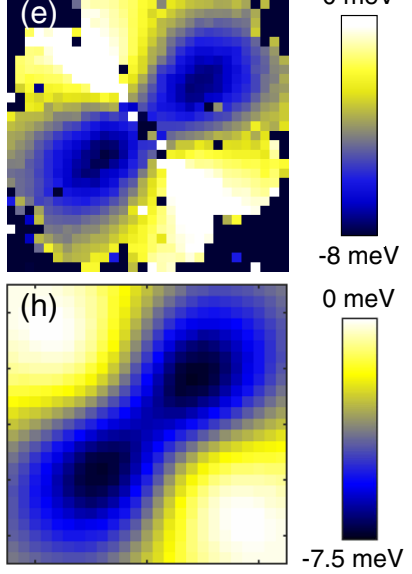

Charge density difference

(k)

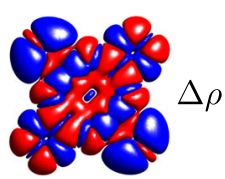

FIG. 3. HRFM mapping of a type- $1 \mathrm{H}_{2} \mathrm{Pc}$ molecule. (a) Constant-height STM current image $\left(V=-2.5 \mathrm{~V}, 2.5 \times 2.5 \mathrm{~nm}^{2}\right.$, $I_{\max }=101 \mathrm{pA}$ ) of a type- $1 \mathrm{H}_{2}$ Pc molecule acquired with an open feedback loop (constant height). (b) Two-dimensional representation of successive STML spectra acquired for STM tip positions along the white arrow in panel (a). The STML spectrum corresponding to the $y$ coordinate of the STM tip marked by the dashed white arrow is plotted as a yellow curve. (c) Photon-intensity, adapted by permission from Springer Nature [42], (d) linewidth, and (e) line-shift maps of the $Q_{x 1}$ exciton reconstructed from STML spectra acquired for each pixel of the map in panel (a). The integration range (1.797-1.813 eV) is indicated by white dashed lines in panel (b). Theory maps of (f) the photon intensity, $(\mathrm{g})$ linewidth, and $(\mathrm{h})$ line shift assuming a 1.2-nm gap between the tip and the substrate and considering a picocavity tip. We present the theory maps consistently with the experiment and measure the line shift relative to the maximal value of the exciton energy (the line shift thus appears to be negative). The molecule is positioned $0.5 \mathrm{~nm}$ above the silver substrate (see the configuration in Fig. 6 in Appendix A). The geometry of the molecule is shown in panel (i) together with (j) the transition charge density and (k) the charge density difference. The maps, the geometry, and the charge densities are shown at the same scale. 
Figure 3(b) is a 2D representation of successive spectra acquired along the vertical arrow in Figure 3(a), where the $Q_{x 1}$ and $Q_{x 2}$ contributions can be identified. Similarly to what was reported in Fig. 1(e), the intensity, energy position, and width of the fluorescence lines vary as a function of the lateral position of the tip with respect to the molecule. As the energy separation between the $Q_{x 1}$ and $Q_{x 2}$ lines of type-1 molecules is substantially larger than their line shifts, the analysis of one peak is not perturbed by the presence of the other. In Fig. 3(c)-3(e), we show the position-dependent maps of the $Q_{x 1}$ line characteristics recorded simultaneously with the current map in Fig. 3(a). The spectral range of the emission is marked by the white dashed lines in Fig. 3(b). In the photon-intensity map [Fig. 3(c)], one first identifies eight lobes that are characteristic of the highest occupied molecular orbital (HOMO) of the molecule. One also notices a dark node that crosses the molecule from the top-left to the bottomright corner of the photon-intensity map in Fig. 3(c). In Figs. 3(d) and 3(e), we display the evolution of the linewidth and the energy of the $Q_{x 1}$ exciton, respectively, for each position of the tip with respect to the molecule. These images reveal similar two-lobe patterns oriented along the $Q_{x 1}$ transition dipole moment of the molecule, where the width of the emission line is broader and the line is redshifted compared to spectra recorded aside from the lobes. However, while these lobes appear rather homogeneous and round in the linewidth map, they adopt a more elongated shape in the line-shift one. Similar data, tilted by $90^{\circ}$, are recorded for the $Q_{x 2}$ contribution (see Appendix E).

To interpret these results, we apply our theoretical model considering the picocavity formed between the silver substrate and an atomic-scale protrusion on the silver STM tip (see Appendix A) and calculate constant-height maps of the light intensity emitted by the electrically pumped molecule [Fig. 3(f)], plasmon-induced linewidth [Fig. 3(g)], and line shift [Fig. 3(h)]. The theoretically calculated maps are in excellent agreement with the experiment. As we discussed in Ref. [42], in the intensity maps [Figs. 3(c) and 3(f)], the information about the plasmonexciton interaction is inherently convoluted with experimental features arising from the electron tunneling, which are proportional to the density of states associated with the $\mathrm{HOMO}$ of the $\mathrm{H}_{2} \mathrm{Pc}$ molecule. Our theoretical Purcell map reproduces both the pattern and the amplitude of the changes of our experimental linewidth maps. Based on this agreement, we attribute the linewidth maps in Figs. 3(d) and 3(g) to the changes of the chromophore's excited-state lifetime as a function of the tip position. The linewidth maps can therefore be interpreted as an artifactfree optical image of the chromophore. Finally, the maps of the relative line shifts shown in Figs. 3(e) and 3(h) reveal an elongated two-lobe pattern featuring the same symmetry as the one obtained in Figs. 3(d) and 3(g). However, in this map, the electrostatic and electrodynamic contributions to the exciton energy shift are entangled.

To further elucidate the physical effects underlying the experimental and theoretical maps in Fig. 3, we analyze the respective role of the plasmonic Purcell effect, the Lamb shift, and the Stark effect for both a sharp picocavity tip [Figs. 4(a)-4(c)] and a blunt tip missing the sharp protrusion [Figs. 4(d)-4(f)]. The line broadening due to the Purcell effect [Figs. 4(a) and 4(d)] features the twolobe structure for both tip shapes but is considerably delocalized when the blunt tip is considered. This points towards the importance of the picocavity in achieving
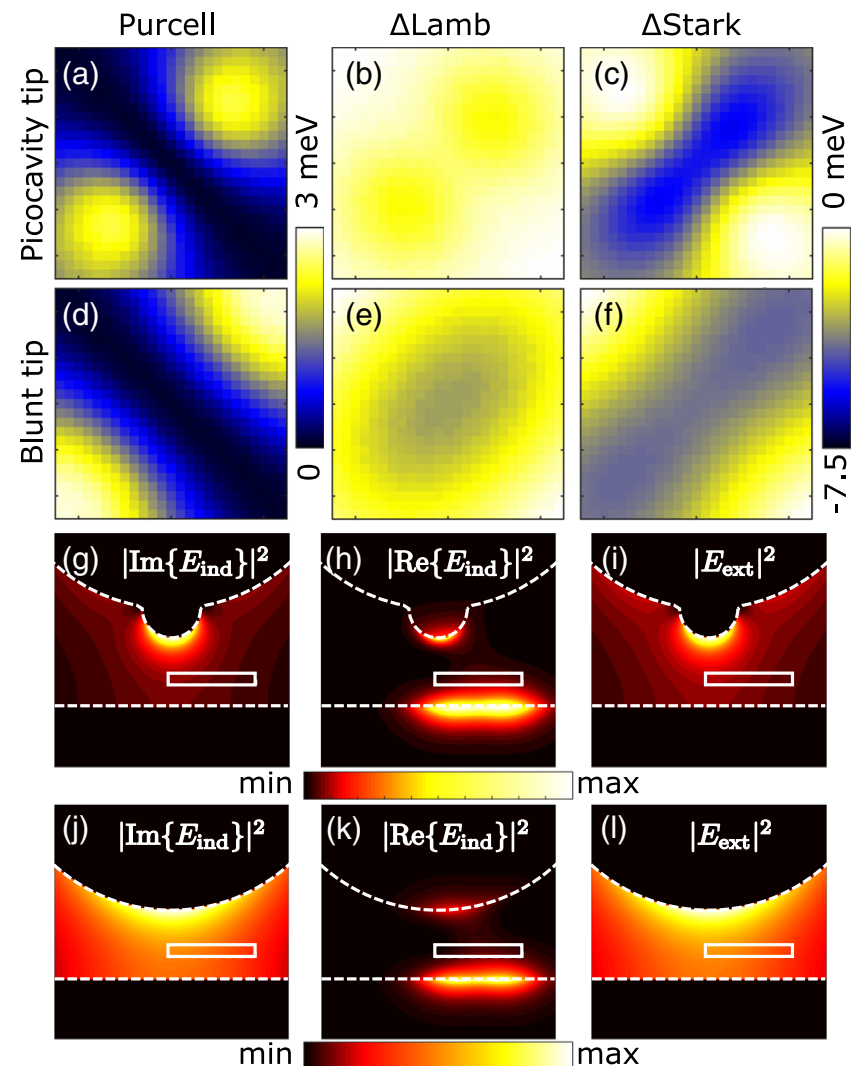

FIG. 4. Dissecting the line broadening and line shift of a singlemolecule exciton in a STM plasmonic cavity. We show the theoretical maps of $(a, d)$ the linewidth, and the variation of $(b, e)$ the Lamb shift and (c,f) the Stark shift induced in (a)-(c) the plasmonic picocavity and (d)-(f) the cavity formed between the blunt tip and the substrate. The values of the shifts are displayed in relative units with respect to the maximal value obtained in the maps. The size of the mapped area is $2.5 \times 2.5 \mathrm{~nm}^{2}$. The color scales in panels $(\mathrm{a}, \mathrm{d})$ and panels $(\mathrm{b}, \mathrm{c}, \mathrm{e}, \mathrm{f})$, respectively, are identical. (g,j) $\left|\operatorname{Im}\left\{\mathbf{E}_{\text {ind }}\right\}\right|^{2}$ and (h,k) $\left|\operatorname{Re}\left\{\mathbf{E}_{\text {ind }}\right\}\right|^{2}$ with $\mathbf{E}_{\text {ind }}=$ $-\nabla \phi_{\text {ind }}$. The induced fields are generated by the $Q_{x}$ exciton of a molecule whose position is marked by the white dashed rectangle. Panels (i,l) show $\left|\mathbf{E}_{\text {exc }}\right|^{2}=\left|\nabla \phi_{\text {ext }}\right|^{2}$. Calculations in panels $(\mathrm{g}, \mathrm{h}, \mathrm{i})$ and $(\mathrm{j}, \mathrm{k}, \mathrm{l})$ have been done for the picocavity tip and the blunt tip, respectively. All the fields are displayed in the plane $y=0$ intersecting the tip axis. The size of the displayed region is $4 \times 4 \mathrm{~nm}^{2}$. 
HRFM. In Figs. 4(b) and 4(e) and Figs. 4(c) and 4(f), we show the Lamb and Stark contributions to the relative lineshift maps, respectively. For the picocavity tip, the Lamb shift map features a faint two-lobe pattern, whereas the Stark shift map reports a more elongated structure that strongly resembles the experimental data. These data also reveal the smaller magnitude of the Lamb shift variations and indicate the prevalence of the Stark effect in the lineshift map. On the other hand, when the blunt tip is considered, the Lamb and Stark shift variations are of more comparable amplitude. As in the case of the Purcell effect, their maps in Figs. 4(e) and 4(f) reveal considerably less-resolved patterns than for the picocavity tip, where the elongated two-lobe structures cannot be recognized anymore.

Despite both being the result of the plasmon-exciton interaction, the patterns observed in the maps of the Lamb shift are qualitatively different from the maps of the linewidth. This is especially apparent for the blunt tip. The origin of this effect can be understood from the spatial distribution of the imaginary part of the induced plasmonic field in the cavity $\left(\left|\operatorname{Im}\left\{\mathbf{E}_{\text {ind }}\right\}\right|^{2}\right.$, with $\left.\mathbf{E}_{\text {ind }}=-\nabla \phi_{\text {ind }}\right)$ responsible for the line broadening [Figs. 4(g) and 4(j)] and its real part $\left(\left|\operatorname{Re}\left\{\mathbf{E}_{\text {ind }}\right\}\right|^{2}\right)$ responsible for the Lamb shift [Figs. 4(h) and 4(k)]. We consider the picocavity tip in Figs. 4(g) and 4(h) and the blunt tip in Figs. 4(j) and 4(k). The spatial distribution of the imaginary part of the induced plasmonic field corresponds to the field intensity generated by the dipolar plasmonic mode whose dipolar moment is oriented along the tip long axis $z$ perpendicular to the substrate. This plasmon mode is tuned to be resonant with the exciton by adjusting the tip geometry as detailed in Appendix A. This field distribution is an intrinsic property of the plasmonic picocavity and is therefore independent of the position of the source molecule. This single resonant dipolar plasmonic mode thus dominantly contributes to the Purcell effect and in turn mediates the far-field excitonic radiation recorded in STML. On the contrary, the real part of the induced field is strongly concentrated on the metal surfaces closely surrounding the molecule (see the bright spot on the metal surfaces below and above the molecule) and depends strongly on the molecular position (marked by the white rectangle). This localization is caused by the bluedetuned, higher-order, surface plasmon modes acting collectively as a plasmonic pseudomode that redshifts the excitonic peak $[60,61]$. The role of the picocavity, in this case, is to further localize the plasmonic response to the protrusion, which is thus sensitive to the spatial variation of the excitonic transition-charge density, highlighting that the chromophore cannot be considered as a simple point dipole $[52,61]$. Finally, $\phi_{\text {ext }}$ features a strong gradient (electric field) $\mathbf{E}_{\mathrm{ext}}=-\nabla \phi_{\mathrm{ext}}$ in the $z$ direction due to the bias voltage. We show the magnitude of the electric field in Fig. 4(i) for the picocavity tip and in Fig. 4(1) for the blunt tip. The protrusion of the picocavity tip additionally generates a strong gradient of $\phi_{\mathrm{ext}}$ in the plane of the substrate, which is significant even across the geometrical extent of the molecule (about $1.5 \mathrm{~nm}$ ). It is this spatial inhomogeneity that results in the strong dependence of the dc Stark shift on the relative position of the molecule with respect to the tip.

Overall, the linewidth and line-shift maps have a welldefined meaning in terms of the local properties of the molecules. As we detail in Appendix F, the linewidth maps are sensitive to the distribution of the excitonic transitioncharge density $\rho_{\text {eg }}$ [shown in Fig. 3(j)] and, in the case of the exciton $Q_{x}$ of $\mathrm{H}_{2} \mathrm{Pc}$, can be understood as images of the position and orientation of the excitonic transition dipole moment. More concretely, their spatial distribution originates from the convolution of the potential $\phi_{\text {dip }} \sim \operatorname{Im}\left\{\phi_{\text {ind }}\right\}$ of the dipolar plasmonic mode of the tip and the transition charge density. In the hypothetical limiting case of a perfectly localized plasmonic response (vanishing mode volume of the dipolar plasmon), these maps could be interpreted as maps of $\left|\rho_{\text {eg }}\right|^{2}$, in analogy with the interpretation of standard STM images within the Tersoff-Hamann approximation [62].

Regarding the line-shift maps, in the case of a dominant linear Stark effect [48], we can attribute their spatial distribution predominantly to the redistribution of electrons in the molecule associated with the excitonic transition. This redistribution is represented by the charge density difference $\Delta \rho$ [shown in Fig. 3(k)]. The linewidth map combined with the line-shift map therefore provides complementary information about the dynamical charge oscillation and static charge redistribution associated with the molecular exciton, respectively.

\section{HRFM OF TYPE 2 MOLECULE}

Having fully addressed the nature of the contrasts in HRFM maps of a type- 1 molecule, we eventually apply this approach to a $\mathrm{H}_{2} \mathrm{Pc}$ molecule of type 2 [Fig. 5(a)] that displays a smaller energy separation between the $Q_{x 1}$ and $Q_{x 2}$ lines. Figure 5(b) is the 2D representation of successive spectra acquired along the vertical arrow in Figure 5(a), analogous to that in Fig. 3(b) for the type-1 molecule. The yellow curve overlaid on the plot represents the STML spectrum indicated by a short white arrow, where $Q_{x 1}$ and $Q_{x 2}$ contributions are identified. Similarly to what is reported for the type-1 molecule, the 2D plot in Fig. 5(b) reveals an energy shift of the $Q_{x 1}$ and $Q_{x 2}$ lines as a function of the tip position. However, as the energy separation between the $Q_{x 1}$ and $Q_{x 2}$ lines is smaller for the type-2 molecule, a configuration is reached (white dotted line, $y \approx 1.1 \mathrm{~nm}$ ) where the two contributions are degenerate. For larger and smaller values of $y$, the two spectral contributions can be separated again. Following an approach similar to that used in Fig. 3, in Figs. 5(c) and 5(d), we generated two line-shift maps by estimating the energy of the intensity maxima in the red and blue energy windows overlaid on Fig. 5(b). In this case, one assumes that the energy of the $Q_{x 1}$ 

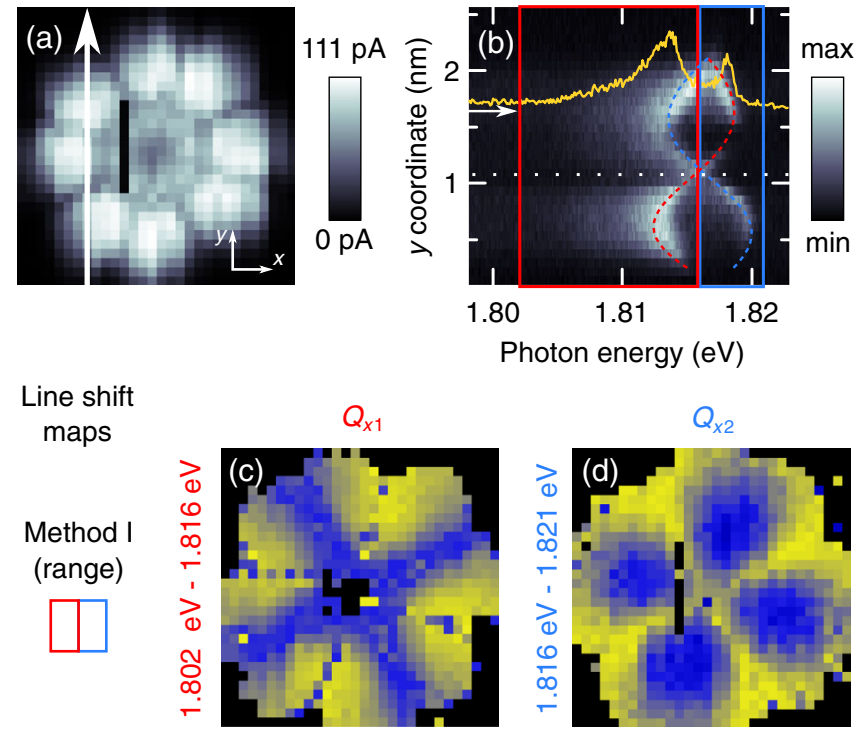

Method II (following the peak)

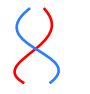

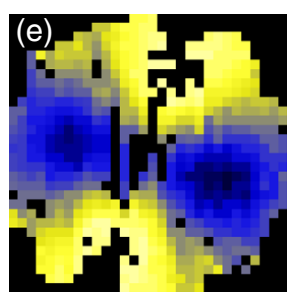

$0 \mathrm{meV}$

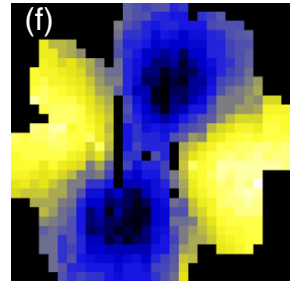

$-8.7 \mathrm{meV}$
FIG. 5. HRFM mapping of a type- $2 \mathrm{H}_{2} \mathrm{Pc}$ molecule. (a) Constant-height STM current image $\left(V=-2.5 \mathrm{~V}, 2.6 \times 2.6 \mathrm{~nm}^{2}\right.$, $I_{\max }=111 \mathrm{pA}$ ) of a type- $2 \mathrm{H}_{2} \mathrm{Pc}$ molecule acquired with an open feedback loop. (b) Two-dimensional representation of successive STML spectra acquired for STM tip positions along the white arrow in panel (a). A spectrum corresponding to the STM tip position marked by a short white arrow is plotted in yellow. (c)(f) HRFM line-shift maps of $Q_{x 1}(\mathrm{c}, \mathrm{e})$ and $Q_{x 2}(\mathrm{~d}, \mathrm{f})$ acquired for each pixel of the map in panel (a). The maps in panels $(c, d)$ are obtained by selecting a range defined by the colored boxes in panel (b). The maps in panels (e,f) are obtained by following the given $Q_{x}$ exciton (dashed colored lines). Note the common color scale for all line-shift maps.

line ( $Q_{x 2}$ line) in Fig. 5(b) is always lower (higher) than $1.816 \mathrm{eV}$, the energy at which the two peaks appear degenerate. In contrast with the maps obtained on type-1 $\mathrm{H}_{2} \mathrm{Pc}$, the line-shift maps reveal two different patterns of an unexpected fourfold symmetry. We also generated line-shift maps assuming that the $Q_{x 1}$ line ( $Q_{x 2}$ line) keeps shifting to higher (lower) energy after crossing the position corresponding to degenerate $Q_{x 1}$ and $Q_{x 2}$ contributions [marked by a dashed line in Fig. 5(b)]. This means considering that the energy of the $Q_{x 1}$ line ( $Q_{x 2}$ line) follows the red (blue) dashed curve. Line-shift maps generated with this method reveal the characteristic twofold symmetry patterns, tilted $90^{\circ}$ from each other, expected for the $Q_{x 1}$ and $Q_{x 2}$ contributions. From this analysis, we conclude that type-2 molecules naturally exhibit degenerate $Q_{x 1}$ and $Q_{x 2}$ transitions, a degeneracy that is lifted by the coupling of the tip with each of the two excited states whose strengths vary as a function of the lateral tip position.

\section{CONCLUSIONS AND OUTLOOK}

In this paper, we decipher the role played by electrostatic and electromagnetic interactions contributing to the unmatched spatial resolution obtained in STM-induced luminescence measurements of single molecules. This enables realizing hyper-resolved fluorescence mapping of two quantities: the width and the shift of fluorescence lines, which, in contrast to more standard intensity maps, provide information completely free of features related to the electron excitation efficiency. These line-shift and linewidth maps unravel the interaction between the excited states of a chromophore with the electrodynamic fields confined at the plasmonic picocavity, revealing the emergence of the Purcell, Lamb, and Stark effects. Their respective impact on the fluorescence maps is dissected with an approach that combines $a b$ initio and electrodynamical calculations.

We foresee that these approaches may be used to reveal new characteristics of molecular systems studied in former STML works discussing line intensity mapping, in particular, those where emission lines are very close in energy (as in the case of type-2 molecules). In addition to the information provided by standard STM, the HRFM photonintensity maps reflect the microscopic mechanism of electrical exciton pumping [42], the maps of linewidths reveal optical properties of the exciton, and the Stark shift maps probe the redistribution of the electron charge between the ground and the excited state and can be well adapted to the study of molecular charge-transfer excitons. This strong Stark effect can be further employed to either gate the energy of individual excitons, for example, in coupled multimolecular architectures [33,34,45], or sense local electrostatic fields. Similarly, sensing of the electromagnetic environment can be obtained by monitoring the Purcell effect. A microscopically controlled Stark shift of excitons has also been proposed to be at the core of molecular optomechanical devices [63] and quantum transducers between superconducting qubits and optical photons [64], or demonstrated to control strong coupling [65]. The tool set provided by the modalities of STML demonstrated in this paper is therefore particularly suited to study the basic building blocks of next-generation, organic lightemitting diodes; organic solar cells; or even molecular sources of nonclassical light and molecular quantum technologies, in general [6].

\section{METHODS}

The STM data were acquired with a low-temperature (4.5-K) ultrahigh-vacuum Omicron setup adapted to detect 
the light emitted at the tip-sample junction. The optical detection setup is composed of a spectrograph combined with a charged-coupled device (CCD) camera, and it provides a spectral resolution of approximately $0.2 \mathrm{~nm}$ [42]. Tungsten STM tips were introduced in the sample to cover them with silver to tune their plasmonic response to be resonant with the exciton, as presented in Appendix G. The $\operatorname{Ag}(111)$ substrates were cleaned with successive argon-ion sputtering and annealing cycles. An approximately 0.5 monolayer of $\mathrm{NaCl}$ was sublimed on $\mathrm{Ag}(111)$ kept at room temperature, forming square bilayers and trilayers after mild postannealing. Free-base phthalocyanine $\left(\mathrm{H}_{2} \mathrm{Pc}\right)$ molecules were evaporated on the cold (approximately $5 \mathrm{~K}$ ) $\mathrm{NaCl} / \mathrm{Ag}(111)$ sample in the STM chamber. As the acquisition of each optical spectrum of the HRFM maps requires a long accumulation time, an atomic tracking procedure is used to correct the $x, y, z$ position of the STM tip between the acquisition of each pixel, ensuring a full correction of residual thermal drifts that may have detrimental effects on STM measurements requiring a long acquisition time.

\section{ACKNOWLEDGMENTS}

The authors thank Virginie Speisser for technical support and Alex Boeglin and Hervé Bulou for discussions. This project has received funding from the European Research Council (ERC) under the European Union's Horizon 2020 research and innovation program (Grant Agreement No. 771850) and the European Union's Horizon 2020 research and innovation program under the Marie Skłodowska-Curie Grant Agreement No. 894434. The Labex NIE (Contract No. ANR-11-LABX-0058_NIE), the International Center for Frontier Research in Chemistry (FRC), the Spanish Ministry of Science (Project No. PID2019-107432 GB-I00), and the Department of Education of the Basque Government (Project No. IT116419) are acknowledged for financial support.

\section{APPENDIX A: THEORETICAL MODELING OF THE LAMB, STARK, AND PURCELL EFFECTS IN A PLASMONIC PICOCAVITY}

The electronic structure of a free-base phthalocyanine is approximated as an electronic two-level system composed of electronic ground $|\mathrm{g}\rangle$ and excited $|\mathrm{e}\rangle$ states. The excited electronic state in this case represents the $Q_{x}$ electronic transition of the molecule. We assume that the $90^{\circ}$ tilted exciton of the molecule, $Q_{y}$, is sufficiently energetically separated from the $Q_{x}$ excitonic transition so that neither the plasmonic interactions nor the Stark effect cause significant mixing of the two. The molecular excited and ground electronic states interact (i) with the static potential $\phi_{\text {ext }}(\mathbf{r})$ due to the bias voltage applied between the tip and the substrate, and (ii) with the dynamical plasmonic potential $\phi_{\text {ind }}$ induced at the junction between the tip and substrate electrodes via their induced charge density. The electron charge density is represented by the operator $\hat{\rho}(\mathbf{r})$, which can be expressed in the second quantization as

$$
\hat{\rho}(\mathbf{r})=\sum_{i j, \alpha} \rho_{i j}(\mathbf{r}) \hat{c}_{i \alpha}^{\dagger} \hat{c}_{j \alpha},
$$

where $\hat{c}_{i \alpha}\left(\hat{c}_{i \alpha}^{\dagger}\right)$ are the fermionic single-particle annihilation (creation) operators with the indices $i, j$ running over spatial orbitals and $\alpha$ running over the spin indices $\uparrow$ and $\downarrow$. The densities $\rho_{i j}(\mathbf{r})$ are defined as $\rho_{i j}(\mathbf{r})=-|\mathrm{e}| \psi_{i}(\mathbf{r})^{*} \psi_{j}(\mathbf{r})$, with $\psi_{i}(\mathbf{r})$ being the respective single-electron spatial orbitals and e the electron charge.

To calculate the molecular ground and excited states, we use the linear-response, time-dependent, density functional theory with the B3LYP functional and $6-31 \mathrm{~g} *$ basis set, as implemented in NWChem [57]. We obtain the excitations in the Tamm-Dancoff (configuration interaction singles, CIS) approximation. In this approximation, the excited states are defined as linear combinations of electron-hole pairs created on top of the filled Fermi sea defined by the ground state:

$$
|\mathrm{g}\rangle=\prod_{a \in \text { occup. }} \hat{c}_{a \uparrow}^{\dagger} \prod_{a \in \text { occup. }} \hat{c}_{a \downarrow}^{\dagger}|0\rangle,
$$

where $|0\rangle$ is the empty vacuum state. Furthermore, we use the convention that $a, b, \ldots$ run over the occupied states of the ground state, and $i, j, \ldots$ run over the unoccupied states. The excited state $|e\rangle$ is generated from the ground state in the Tamm-Dancoff approximation as

$$
|\mathrm{e}\rangle=\sum_{i a \alpha} C_{i a, \alpha} \hat{c}_{i \alpha}^{\dagger} \hat{c}_{a \alpha}|\mathrm{g}\rangle .
$$

Here, $C_{i a, \alpha}$ are coefficients fulfilling $\sum_{i a \alpha}\left|C_{i a, \alpha}\right|^{2}=1$, and for singlet excitations, we have $C_{i a, \downarrow}=C_{i a, \uparrow}=C_{i a}$.

\section{The dc Stark effect in plasmonic picocavities}

The dc Stark effect commonly refers to the shift of the energies of quantum states of a system exposed to an external static electric field. The interaction energy of this external field with the electron density $\rho(\mathbf{r})$ of the system can be expressed by the Hamiltonian

$$
\hat{H}_{\text {Stark }}=\iiint \hat{\rho}(\mathbf{r}) \phi_{\text {ext }}(\mathbf{r}) d^{3} \mathbf{r} .
$$

The external potential $\phi_{\text {ext }}$ usually acts as a perturbation to the potential of the quantum system and thus leads to energy shifts that can be obtained using the RayleighSchrödinger perturbation theory. Using Eq. (A4), we calculate the Stark shift experienced by the electronic excitation between the electronic ground state $|\mathrm{g}\rangle$ of energy $E_{\mathrm{g}}$ and the excited state $|\mathrm{e}\rangle$ of energy $E_{\mathrm{e}}$ using the first order 
of perturbation theory-considering, therefore, the linear component of the Stark shift as

$$
\delta E_{\mathrm{St}}=\left\langle\mathrm{e}\left|\hat{H}_{\text {Stark }}\right| \mathrm{e}\right\rangle-\left\langle\mathrm{g}\left|\hat{H}_{\mathrm{Stark}}\right| \mathrm{g}\right\rangle=\int \Delta \rho(\mathbf{r}) \phi_{\mathrm{ext}}(\mathbf{r}) d^{3} \mathbf{r},
$$

with $\Delta \rho(\mathbf{r})=\rho_{\mathrm{e}}(\mathbf{r})-\rho_{\mathrm{g}}(\mathbf{r})$. The experimental evidence [Fig. 2(e)] suggests that the peak shift scales linearly with the external voltage, and the higher-order corrections therefore play a marginal role [48]. Thus, we model only the linear component of the Stark shift.

We calculate the charge density difference $\Delta \rho$ by using the definition of the electronic excited state [Eq. (A3)] and the definition of the density operator [Eq. (A1)]. The electron density of $|\mathrm{g}\rangle$ is

$$
\rho_{\mathrm{g}}=\langle\mathrm{g}|\hat{\rho}| \mathrm{g}\rangle=\sum_{a, \alpha} \rho_{a a}(\mathbf{r})=2 \sum_{a} \rho_{a a}(\mathbf{r})
$$

The electron density of the excited state can be described as

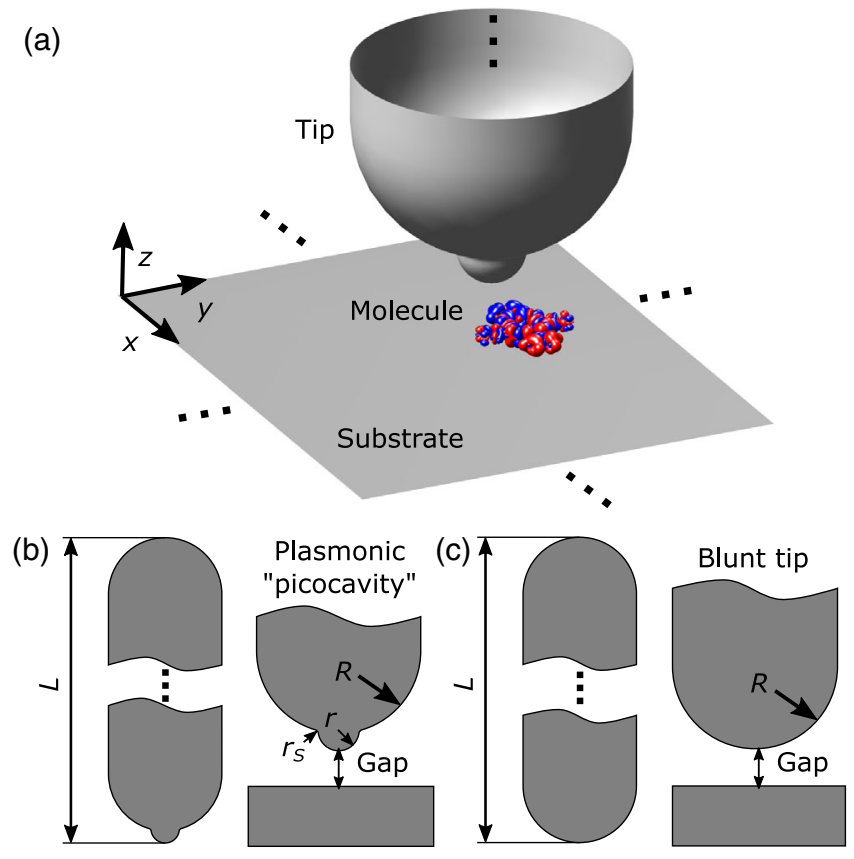

FIG. 6. Geometry of the plasmonic cavities used to model the plasmonic response in STML. (a) Schematic representation of the setup containing a tip, a substrate, and a molecule (represented by the transition charge density of $Q_{x}$ ) placed into the gap between the substrate and the tip. (b) Geometry of the picocavity tip of length $L=30.86 \mathrm{~nm}$. The radius of the cylindrical capsule with hemispherical caps is $R=3 \mathrm{~nm}$. The atomistic protrusion defining the picocavity is modeled using $r=0.5 \mathrm{~nm}$, $r_{\mathrm{s}}=0.1 \mathrm{~nm}$. (c) Blunt tip modeled as a cylindrical capsule of radius $R=3 \mathrm{~nm}$ and length $L=29.58 \mathrm{~nm}$. The molecule is placed $0.5 \mathrm{~nm}$ above the substrate, and the gap size is varied.

$$
\begin{aligned}
\rho_{\mathrm{e}}=\langle\mathrm{e}|\hat{\rho}| \mathrm{e}\rangle= & \sum_{a, \alpha} \rho_{a a}(\mathbf{r})+\sum_{i j a, \alpha} C_{i a, \alpha}^{*} C_{j a, \alpha} \rho_{i j}(\mathbf{r}) \\
& -\sum_{i a b, \alpha} C_{i a, \alpha}^{*} C_{i b, \alpha} \rho_{b a}(\mathbf{r}) .
\end{aligned}
$$

The charge density difference $\Delta \rho(\mathbf{r})$ therefore becomes

$$
\begin{aligned}
\Delta \rho(\mathbf{r}) & =\rho_{\mathrm{e}}(\mathbf{r})-\rho_{\mathrm{g}}(\mathbf{r}) \\
& =\sum_{i j a, \alpha} C_{i a, \alpha}^{*} C_{j a, \alpha} \rho_{i j}(\mathbf{r})-\sum_{i a b, \alpha} C_{i a, \alpha}^{*} C_{i b, \alpha} \rho_{b a}(\mathbf{r}) .
\end{aligned}
$$

We calculate the external potential $\phi_{\text {ext }}$ due to the bias voltage applied between the tip and the substrate using the finite-element method as implemented in the ac/dc module of COMSOL Multiphysics. The geometry used for the calculation is schematically shown in Fig. 6. The tip is modeled as an elongated cylinder with hemispherical caps whose bottom surface additionally supports a sharp protrusion of radius $R=0.5 \mathrm{~nm}$, conservatively accounting for the atomic sharpness of the tip. In the practical implementation, we extract the molecular orbitals $\psi_{i}(\mathbf{r})$ in the form of Gaussian cube files and use them as inputs for further postprocessing. In the sum given by Eq. (A7), we consider the dominant coefficients $\sqrt{2}\left|C_{i a, \alpha}\right|>0.01$.

\section{The plasmon-induced Lamb shift and Purcell effect}

We describe the plasmon-induced Lamb shift and broadening of the excitonic emission spectral lines of single molecules in STML in a hybrid framework that combines the quantum properties of the molecular emitter with the classical linear-response description of the surface plasmons. The molecular emitter is represented by the positiondependent excitonic transition charge density $\rho_{\text {eg }}(\mathbf{r})$, which acts as the source of a quasistatic electric field that triggers the plasmonic response in the surrounding material. The induced plasmonic potential $\phi_{\text {ind }}(\mathbf{r})$ can then be calculated using the linear-response theory, which can be expressed by defining a general response function $\chi$ of the dielectric environment as follows

$\phi_{\text {ind }}(\mathbf{r}, t)=\int_{-\infty}^{t} d t^{\prime} \iiint d^{3} \mathbf{r}^{\prime} \chi\left(\mathbf{r}, \mathbf{r}^{\prime}, t-t^{\prime}\right) \tilde{\rho}_{\mathrm{eg}}\left(\mathbf{r}^{\prime}, t^{\prime}\right)$.

The response function $\chi$ relates $\tilde{\rho}_{\mathrm{eg}}\left(\mathbf{r}^{\prime}, t^{\prime}\right)$ at position $\mathbf{r}^{\prime}$ and time $t^{\prime}$ with the time-dependent induced plasmonic potential $\phi_{\text {ind }}(\mathbf{r}, t)$ at position $\mathbf{r}$ and time $t$. In the semiclassical approximation, we assume that this potential is related to the mean value of the transition-charge density operator

$$
\tilde{\rho}_{\mathrm{eg}}(\mathbf{r}, t)=\rho_{\mathrm{eg}}(\mathbf{r})\langle\sigma(t)\rangle,
$$

where $\langle\sigma(t)\rangle$ is the mean value of the oscillating lowering operator of the molecular exciton $\sigma=|\mathrm{g}\rangle\langle\mathrm{e}|$, with $|\mathrm{g}\rangle$ the ground and $|e\rangle$ the excited electronic state of the molecule, 
respectively, and $\rho_{\mathrm{eg}}(\mathbf{r})$ is the time-independent distribution of the transition charge density

$$
\rho_{\mathrm{eg}}(\mathbf{r})=\langle\mathrm{g}|\hat{\rho}(\mathbf{r})| \mathrm{e}\rangle=\sum_{i a, \alpha} C_{i a, \alpha} \rho_{i a}(\mathbf{r}) .
$$

In our calculation, $\rho_{\mathrm{eg}}(\mathbf{r})$ is extracted from NWChem in the form of a Gaussian cube file. We note that the transition dipole moment of the molecule $\boldsymbol{\mu}$ is linked to the transition-charge density via $\boldsymbol{\mu}=\iiint \rho_{\mathrm{eg}}(\mathbf{r}) d^{3} \mathbf{r}$. Using the rotating-wave approximation, we can write the effective Hamiltonian $H_{\text {ind }}$ of the molecule under the influence of the induced field as

$$
H_{\text {ind }}=\iiint d^{3} \mathbf{r} \phi_{\text {ind }}(\mathbf{r}, t) \rho_{\mathrm{eg}}^{*}(\mathbf{r}) \sigma^{\dagger}+\text { H.c. },
$$

where H.c. stands for the Hermitian conjugate. We further assume that the electronic transition in the unperturbed molecule is described by the Hamiltonian $H_{\text {mol }}$

$$
H_{\mathrm{mol}}=\hbar \omega_{\mathrm{eg}} \sigma^{\dagger} \sigma
$$

with $\omega_{\mathrm{eg}}$ being the transition frequency of the unperturbed exciton. Under the influence of $H_{\text {mol }}$, the exciton of the molecule experiences time-harmonic oscillation so that $\langle\sigma(t)\rangle \propto e^{-i \omega_{\mathrm{eg}} t}$. The mean value of the operator $\sigma,\langle\sigma\rangle$, evolving according to $H_{\text {tot }}=H_{\text {mol }}+H_{\text {ind }}$ is thus governed by the linearized equation

$$
\begin{aligned}
\langle\sigma(t)\rangle & =-\mathrm{i} \omega_{\mathrm{eg}}\langle\sigma(t)\rangle-\frac{\mathrm{i}}{\hbar} \iiint d^{3} \mathbf{r} \phi_{\mathrm{ind}}(\mathbf{r}, t) \rho_{\mathrm{eg}}^{*} \\
& =-\mathrm{i} \omega_{\mathrm{eg}}\langle\sigma(t)\rangle-\frac{\mathrm{i}}{\hbar} \int_{-\infty}^{t} d t^{\prime} \iiint d^{3} \mathbf{r} \iiint d^{3} \mathbf{r}^{\prime} \rho_{\mathrm{eg}}^{*}(\mathbf{r}) \chi\left(\mathbf{r}, \mathbf{r}^{\prime}, t-t^{\prime}\right) \rho_{\mathrm{eg}}\left(\mathbf{r}^{\prime}\right)\left\langle\sigma\left(t^{\prime}\right)\right\rangle .
\end{aligned}
$$

Next, we assume that the induced potential acts in a perturbative way and induces only a minor change to the time evolution of $\langle\sigma(t)\rangle$. We therefore evaluate the time integral in the adiabatic approximation

$$
\begin{aligned}
& \int_{-\infty}^{t} d t^{\prime} \iiint d^{3} \mathbf{r} \iiint d^{3} \mathbf{r}^{\prime} \rho_{\mathrm{eg}}^{*}(\mathbf{r}) \chi\left(\mathbf{r}, \mathbf{r}^{\prime}, t-t^{\prime}\right) \rho_{\mathrm{eg}}\left(\mathbf{r}^{\prime}\right)\left\langle\sigma\left(t^{\prime}\right)\right\rangle \\
& \quad \approx\langle\sigma(t)\rangle \int_{-\infty}^{t} d t^{\prime} \iiint d^{3} \mathbf{r} \iiint d^{3} \mathbf{r}^{\prime} \rho_{\mathrm{eg}}^{*}(\mathbf{r}) \chi\left(\mathbf{r}, \mathbf{r}^{\prime}, t-t^{\prime}\right) \rho_{\mathrm{eg}}\left(\mathbf{r}^{\prime}\right) e^{\mathrm{i} \omega_{\mathrm{eg}}\left(t-t^{\prime}\right)} \\
& \quad \approx\langle\sigma(t)\rangle \iiint d^{3} \mathbf{r} \iiint d^{3} \mathbf{r}^{\prime} \rho_{\mathrm{eg}}^{*}(\mathbf{r}) \tilde{\chi}\left(\mathbf{r}, \mathbf{r}^{\prime}, \omega_{\mathrm{eg}}\right) \rho_{\mathrm{eg}}\left(\mathbf{r}^{\prime}\right) \\
& \quad=\langle\sigma(t)\rangle \iiint d^{3} \mathbf{r} \rho_{\mathrm{eg}}^{*}(\mathbf{r}) \phi_{\mathrm{ind}}\left(\mathbf{r}, \omega_{\mathrm{eg}}\right)
\end{aligned}
$$

where we have defined the Fourier transform $\tilde{\chi}$ of the response function $\chi$, and the Fourier transform of the induced potential $\phi_{\text {ind }}\left(\mathbf{r}, \omega_{\text {eg }}\right)$ (i.e., the induced potential calculated in the frequency domain).

The time dependence of the mean value $\langle\sigma\rangle$ thus becomes

$$
\langle\sigma(t)\rangle \approx\langle\sigma(0)\rangle e^{-\mathrm{i}\left[\omega_{\mathrm{eg}}+f\left(\mathbf{R}, \omega_{\mathrm{eg}}\right)\right] t}
$$

with

$$
f\left(\mathbf{R}, \omega_{\mathrm{eg}}\right)=\frac{1}{\hbar} \iiint d^{3} \mathbf{r} \rho_{\mathrm{eg}}^{*}(\mathbf{r}-\mathbf{R}) \phi_{\mathrm{ind}}\left(\mathbf{r}, \omega_{\mathrm{eg}} ; \mathbf{R}\right) .
$$

Here, we have made explicit the dependence of the density $\rho_{\text {eg }}(\mathbf{r}-\mathbf{R})$ and induced potential $\phi_{\text {ind }}\left(\mathbf{r}, \omega_{\text {eg }} ; \mathbf{R}\right)$ on the relative position $\mathbf{R}$ of the molecule with respect to the tip, which we have omitted in the main text and Eqs. (A8) to (A14) for brevity. The spectral response $S_{\mathrm{e}}(\omega)$ of the exciton can be obtained from the quantum regression theorem relating the dynamics of $\langle\sigma(t)\rangle$ to the dynamics of the two-time correlation function $\left\langle\sigma^{\dagger}(0) \sigma(t)\right\rangle \propto\langle\sigma(t)\rangle$ [66], yielding

$$
\begin{aligned}
S_{\mathrm{e}}(\omega) & \propto \operatorname{Re}\left\{\int_{0}^{\infty}\left\langle\sigma^{\dagger}(0) \sigma(t)\right\rangle e^{\mathrm{i} \omega t} d t\right\} \\
& \propto \frac{\frac{\gamma_{\mathrm{eg}}}{2}}{\left(\omega_{\mathrm{eg}}+\delta \omega_{\mathrm{eg}}-\omega\right)^{2}+\left(\frac{\gamma_{\mathrm{eg}}}{2}\right)^{2}} .
\end{aligned}
$$

Here, the frequency shift (Lamb shift) $\delta \omega_{\mathrm{eg}}$ and line broadening (Purcell effect) $\gamma_{\text {eg }}$ induced by the plasmons are related to $f\left(\mathbf{R}, \omega_{\mathrm{eg}}\right)$ as 


$$
\begin{gathered}
\delta \omega_{\mathrm{eg}}(\mathbf{R})=\operatorname{Re}\left\{f\left(\mathbf{R}, \omega_{\mathrm{eg}}\right)\right\}, \\
\gamma_{\mathrm{eg}}(\mathbf{R})=-2 \operatorname{Im}\left\{f\left(\mathbf{R}, \omega_{\mathrm{eg}}\right)\right\} .
\end{gathered}
$$

We use the expressions in Eqs. (A18) and (A19) to calculate the Lamb shift and Purcell effect.

Note that in our calculations, we can neglect the role of the tunneling current due to the dynamics of our system. The low-ps excited-state lifetime constitutes a reference point in that respect. Both the tunneling current (the average time between consecutive charges) and injection from the substrate occur either on the order of or slower than $1 \mathrm{~ns}$ [67]. Therefore, a current-excited molecule will decay before another charge interacts with it, and thus the current can be neglected in the model. This assumption also agrees with the experiment, as there is no correlation between the current map [Fig. 3(a)] and the linewidth map [Fig. 3(d)]. We note that the electronic coupling to the underlying metallic substrate [68], despite being reduced by the insulating $\mathrm{NaCl}$ layer, may still play a role in shortening the exciton lifetime. However, this effect would not contribute to the spatial variations appearing in the linewidth maps.

\section{Quasistatic calculation of the plasmonic induced electric potential}

We calculate the induced potential in the quasistatic approximation using the finite-element method implemented in the ac/dc module of COMSOL Multiphysics and describe the dielectric response of the silver tip and the substrate using the silver dielectric function obtained from Ref. [69]. In the calculations, we simplify the geometry of the tip as shown in Fig. 6. The geometry of the tip is tailored to provide a dipole plasmonic resonance along the long axis, $z$, of the tip resonant with the molecular exciton. In this way, we account for the resonant plasmonic effects associated with the bright plasmonic mode of the tip, which efficiently transmit the excitonic emission into the far field. We import the excitonic transition charge density from the TD-DFT calculation and use it as a source of the classical potential generated in the geometry defined by the tip and the substrate. We impose zero potential on the boundary of the simulation domain ensuring convergence of the results.

\section{Spectral response of the plasmonic cavity}

We study the spectral response of the plasmonic picocavity and the cavity formed by the blunt tip and the substrate shown in Fig. 6. To that end, we place the molecule at the position $[x, y]=[0.69,0.19] \mathrm{nm}$ with respect to the tip and $0.5 \mathrm{~nm}$ above the substrate and fix the gap size to $1.2 \mathrm{~nm}$. We consider that the plasmonic response is stimulated by the $Q_{x}$ exciton and we treat the frequency of the exciton as a free parameter. For each
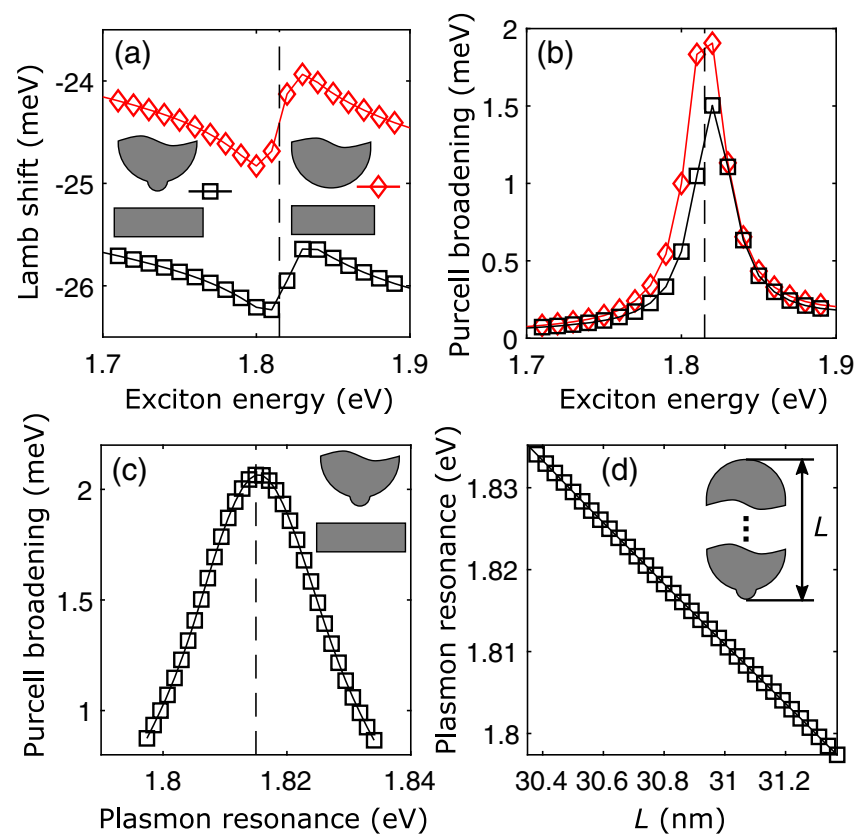

FIG. 7. Spectral dependence of the Lamb shift and the Purcell effect. (a) Lamb shift calculated as a function of the exciton frequency for the picocavity (black squares) and the blunt tip (red diamonds). (b) Broadening of the excitonic peak due to the Purcell effect for the picocavity (black squares) and the blunt tip (red diamonds). The dashed black line marks the exciton energy of $1.815 \mathrm{eV}$ considered for the calculation of the spatial dependence of the Lamb shift and the Purcell effect in this work. (c) Dependence of the Purcell broadening on the plasmon resonance of the tip. The tip is positioned as in panels $(\mathrm{a}, \mathrm{b})$, and the total length $L$ of the tip is varied to tune the resonance of the dipolar plasmon. The exciton energy is fixed at $1.815 \mathrm{eV}$. The plasmon resonance of the tip as a function of the tip length $L$ is shown in panel (d).

frequency of the exciton, we calculate the plasmonic Lamb shift and Purcell effect (broadening) and plot the results in Fig. 7. Figure 7(a) shows the spectral dependence of the Lamb shift, and Fig. 7(b) depicts it for the broadening of the exciton peak due to the plasmonic Purcell effect. The results for the picocavity are shown as red diamonds and for the blunt tip as black squares. A resonance feature is observed for the exciton energy around $1.815 \mathrm{eV}$, which appears as a dip followed by a peak in the Lamb-shift spectra and has the form of a peak in the Purcell-broadening spectra. This spectral feature can be attributed to the longitudinal dipole plasmon resonance of the tip described in the main text. We further notice that the resonance feature in the Lamb-shift spectra is strongly offset by a background shift induced by higher-order plasmon modes, which dominate the overall shift. Furthermore, for the exciton energy corresponding to $1.815 \mathrm{eV}$, the shift caused resonantly by the dipole mode completely vanishes. On the other hand, the spectral response of the Purcell broadening is exclusively formed by the resonance feature caused by 
the interaction of the exciton with the resonant dipole plasmon mode. This supports the analysis introduced in Sec. III.

We use the calculated spectral dependence of the Purcell broadening to extract the information about the coupling strength $g$ between the dipolar plasmon of the tip and the molecular exciton. To do so, we fit the following expression derived for the line broadening [61]

$$
\gamma_{\mathrm{eg}} \approx \frac{g^{2} \gamma_{\mathrm{pl}}}{\left(\omega_{\mathrm{eg}}-\omega_{\mathrm{pl}}\right)^{2}+\left(\gamma_{\mathrm{pl}} / 2\right)^{2}}
$$

This expression is derived in the Jaynes-Cummings approximation assuming that only a single plasmonic mode of damping $\gamma_{\mathrm{pl}}$ and frequency $\omega_{\mathrm{pl}}$ resonantly interacts with the molecular exciton of frequency $\omega_{\mathrm{eg}}$. By fitting the data shown in Fig. 7 , we obtain $\hbar g \approx 4.1 \mathrm{meV}$ for the picocavity and $\hbar g \approx 3.6 \mathrm{meV}$ for the blunt tip.

Finally, in Fig. 7(c), we show the influence of the spectral position of the plasmon resonance on the line broadening of the exciton. To that end, we tune the length of the tip while maintaining the gap size of $1.2 \mathrm{~nm}$. The frequency of the dipole plasmon as a function of the tip length $L$ is shown in Fig. 7(d). The relative position of the molecule with respect to the tip is kept as in Figs. 7(a) and 7(b); however, we now keep the energy of the exciton fixed at $1.815 \mathrm{eV}$. We see that in analogy with Figs. 7(a) and 7(b), the linewidth is showing a resonance profile as the plasmon frequency is tuned and it is maximized when the plasmon resonance coincides with the exciton resonance. This happens approximately for a tip with a length of $L=30.86 \mathrm{~nm}$, which defines the tip geometry considered throughout this work. We note that compared to the experimental spectra (Fig. 13), the theoretically obtained plasmon resonance is sharper. The broader peak in the experiment can be due to the complex geometry of the tip (and hence its complex plasmonic response), whose details are generally unknown; due to the radiation damping of the tip plasmon, which is neglected in our quasistatic calculation; or due to the contact of the silver apex of the tip with its tungsten support. We note that the precise modeling of the tip geometry is a challenging task beyond the scope of the present study. Our model, however, captures the main ingredients required for successful STML mapping: (i) the spectral proximity of a plasmonic resonance of the tip to the resonance energy of the studied exciton and (ii) the sharpness of the tip.

\section{APPENDIX B: LORENTZIAN FITS OF THE $Q_{x 1}$ AND $Q_{x 2}$ FLUORESCENCE LINES}

Figure 8 displays Lorentzian fits to the measured $Q_{x 1}$ and $Q_{x 2}$ lines. While these fits reasonably reproduce the light intensities, the peak widths, and the peak positions of the two contributions, they fail to capture the asymmetric nature of

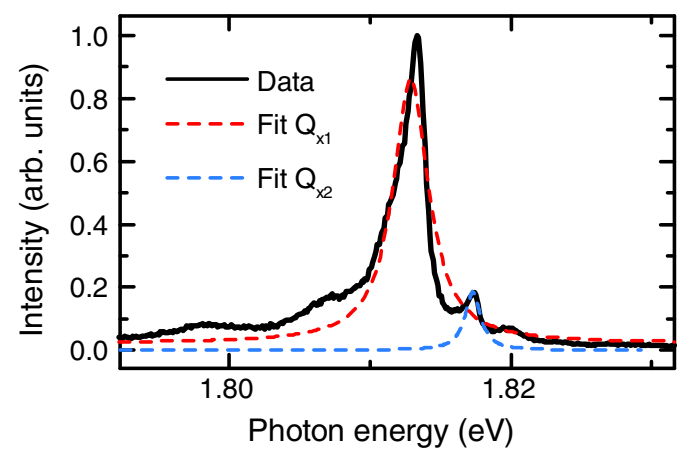

FIG. 8. Typical STML spectrum acquired for a $\mathrm{H}_{2} \mathrm{Pc}$ molecule of type 2, with Lorentzian fits adjusted to the $Q_{x 1}$ and $Q_{x 2}$ lines.

the lines and therefore contribute to the mismatch between the theory and experiment observed in Fig. 2(f). As stated in the main text, this asymmetry and/or broadening may find its origin in different interactions (exciton-vibrations, exciton-phonon, exciton-plasmon), which we discuss in detail below.

One of the effects that may occur in our system involves electronic transitions from vibrationally excited states [48]. In that case, the 0-0 transition may be accompanied by 1-1, 2-2, and similar transitions that have slightly different energies, effectively resulting in a broadening of the spectral line. Each of these transitions would also individually broaden due to the Purcell effect as the tip approaches the molecule. In addition, when the tip moves towards the molecule, the lifetime of the 0-0 transition decreases, which will increase the probability for other $n-n$ transitions to occur due to the breakdown of Kasha's rule. Both of the consequences of the Purcell effect can contribute to the discrepancy between the experiment and theory regarding peak widths. Besides such transitions, one may identify additional spectral features, such as the likely influence of lowenergy vibrations of $\mathrm{H}_{2} \mathrm{Pc}$ [70] or coupling to the $\mathrm{NaCl}$ phonons [71]. Both features appear in the concerned range up to $200 \mathrm{~cm}^{-1}(25 \mathrm{meV}$ ) and can account for the peak broadening and/or asymmetry. Note that precise identification of such mechanisms is beyond the resolution of our experiment. In addition, the coupling between the molecule and its metallic surrounding may also contribute to the modification of the emission line. For instance, the spectral broadening can originate from electronic quenching of the exciton due to the partial overlap of the molecular orbitals with the orbitals of the silver substrate. Furthermore, plasmon-exciton interference mechanisms could result in an intrinsically asymmetric peak (Fano line shape [37,38]).

In the absence of a definitive statement regarding the peak asymmetry and since including effects mentioned above is beyond the scope of our model, we favor adjusting our spectra with simple Lorentzians that do not presume the origin of the phenomena. 


\section{APPENDIX C: STARK AND LAMB SHIFT AS A FUNCTION OF GAP SIZE}

We calculate the Stark shift and the Lamb shift as a function of the gap size between the tip and the substrate and show the result in Fig. 9. In the numerical calculation, we account for the experimentally nonequivalent orientation of $Q_{x 1}$ and $Q_{x 2}$ with respect to the tip by considering two positions of the tip $[x, y]=[0.69,0.19] \mathrm{nm}$ (for $Q_{x 1}$ ) and $[x, y]=[0.19,0.69] \mathrm{nm}$ (for $Q_{x 2}$ ) with respect to the transition charge density of $Q_{x}$ as shown in Fig. 9(a). In Fig. 9(b), we show the numerically calculated total shift of the exciton as a function of the gap between the tip and the substrate for $Q_{x 1}$ (red circles) and $Q_{x 2}$ (blue circles). Notice that in the theory, we only determine the energy shift but not the absolute value of the exciton energy, which is why in Fig. 9(b) we offset the theoretical and experimental data. We add a constant value of $25.5 \mathrm{meV}$ to the numerically calculated shift (the sum of Stark and Lamb shifts). For comparison, we also display the experimental data from Fig. 2(d) of the main text for the type-2 molecule from which we subtract the value of $1.815 \mathrm{eV}$, which we interpret as the energy of the exciton on the substrate, unperturbed by the tip. Finally, we measure the relative distance shown (a)

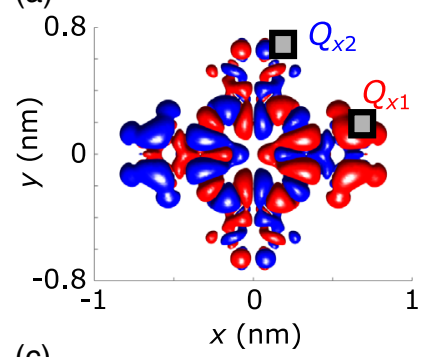

(c)

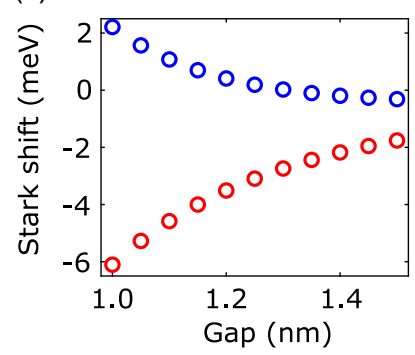

(b)

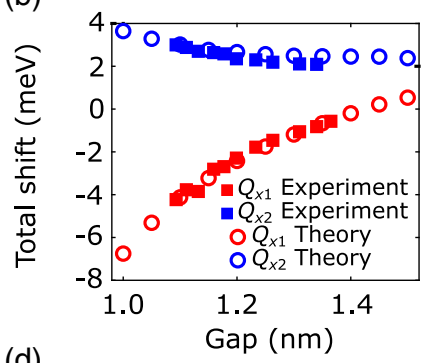

(d)

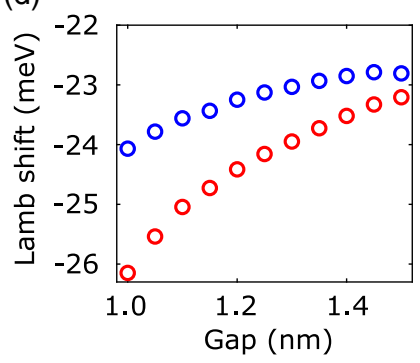

FIG. 9. Excitonic peak shift as a function of tip-substrate gap. (a) Transition charge density of $Q_{x}$ with two squares marking the positions of the tip used to calculate the Stark and the Lamb shift of excitons $Q_{x 1}$ and $Q_{x 2}$. (b) Total peak shift calculated theoretically (circles) displayed together with the experimental data (squares) for $Q_{x 1}$ (red) and $Q_{x 2}$ (blue). For the theoretical data, the origin of the energy scale is offset and corresponds to $-25.5 \mathrm{meV}$ on the absolute scale. An energy of $1.815 \mathrm{eV}$ corresponding to the energy of the unperturbed exciton on the substrate has been subtracted from the experimental data. The numerically calculated contributions to the total shift emerging from the Stark shift and from the Lamb shift are shown in panels (c) and (d), respectively. in Fig. 2(d) with respect to a gap equal to $1.365 \mathrm{~nm}$. The contributions to the total shift originating from the Stark effect and the Lamb shift are shown in Figs. 9(c) and 9(d), respectively. This decomposition shows that the Stark shift gives rise to a decrease of the energy corresponding to $Q_{x 1}$ and an increase of the energy of $Q_{x 2}$ as the gap size is decreased. On the other hand, the Lamb shift leads to lowering of the excitonic energy for both $Q_{x 1}$ and $Q_{x 2}$ with decreasing gap size, as discussed in the main text.

\section{APPENDIX D: PROBING STARK EFFECT AT THE SINGLE-MOLECULE LEVEL}

In Fig. 10, we display normalized STML spectra acquired on the type-2 molecule discussed in Fig. 2(e) for the position marked by a black dot in the STM image in the inset, at a fixed tip-molecule distance (i.e., open feedback loop) and for an increasing bias applied between the STM electrodes. As such, the plasmon-exciton interactions are kept constant, and the spectral shift of the $Q_{x 1}$ and $Q_{x 2}$ STML spectra can be readily associated to changes in the electrical field, i.e., the Stark effect. Here, one observes a subtle redshift (blueshift) of the $Q_{x 1}\left(Q_{x 2}\right)$ line with increasing electric field, an effect that is discussed in Fig. 2(e). Figure 10(b) shows a constant-height scanning tunneling spectroscopy measurement and the corresponding tunneling current recorded with the same tip position as the data in panel (a), showing how the density of states of the molecule changes over the considered voltage range.

\section{APPENDIX E: HRFM MAPPING OF THE $Q_{x 2}$ MODE OF A TYPE-1 $\mathrm{H}_{2}$ Pc MOLECULE}

In Fig. 11, we show the photon-intensity, linewidth, and line-shift maps of the $Q_{x 2}$ line (integration range: 1.821$1.837 \mathrm{eV}$ ) associated to the type- $1 \mathrm{H}_{2} \mathrm{Pc}$ molecule of Fig. 3. As expected, these maps reveal similar patterns to the one
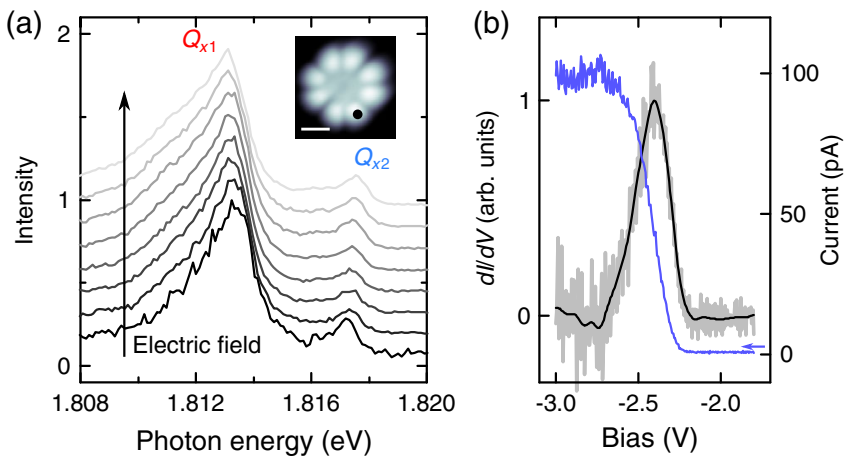

FIG. 10. (a) STML spectra of a type-2 molecule recorded at a fixed tip-sample distance for bias voltages varying from $-2.3 \mathrm{~V}$ (bottom curve) to $-3 \mathrm{~V}$ (top curve) in $0.1 \mathrm{~V}$ increments. Inset: STM image of the $\mathrm{H}_{2} \mathrm{Pc}$ molecule. The measurement position is marked by a dot; $V=-2.5 \mathrm{~V}, I=5 \mathrm{pA}$, and the scale bar is $1 \mathrm{~nm}$. (b) Constant-height scanning tunneling spectroscopy of the $\mathrm{H}_{2} \mathrm{Pc}$ molecule recorded at a position marked in panel (a). 


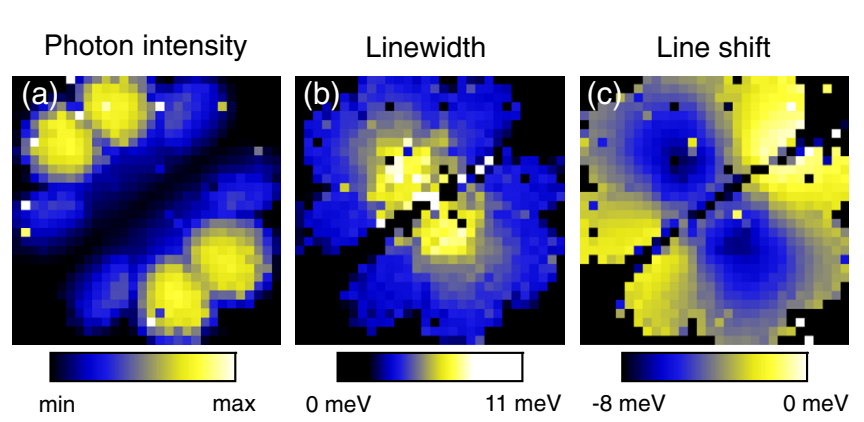

FIG. 11. HRFM mapping of the $Q_{x 2}$ mode of a type- $1 \mathrm{H}_{2} \mathrm{Pc}$ molecule. (a) Photon-intensity, adapted with permission from Ref. [42], Springer Nature Ltd., (b) linewidth, and (c) line-shift maps of the $Q_{x 2}$ line reconstructed from STML spectra acquired for each pixel of the map in Fig. 3(a) of the main text. The integration range is $1.821-1.837 \mathrm{eV}$. Note that, as expected, the observed spatial features are similar to the ones observed for $Q_{x 1}$ in Fig. 3 of the main text but rotated by $90^{\circ}$

of the $Q_{x 1}$ spectral line but tilted by $90^{\circ}$. One also notices that the linewidth maxima and line-shift minima are located slightly closer to the center of the molecule compared to the $Q_{x 1}$ maps, a behavior that we tentatively associate to an environmental effect linked to different molecular dipolesubstrate configurations.

\section{APPENDIX F: INTERPRETING THE LINEWIDTH MAPS}

In Fig. 12, we show the linewidth map calculated using the model described in Appendix A. Here, we show that this linewidth map can be understood as a square modulus of the convolution of the quantized potential $\phi_{\text {dip }}$ of the dipolar plasmonic mode (resonant with the molecular exciton $Q_{x}$ ) and the excitonic transition charge density $\rho_{\text {eg }}$. To prove that, we now approximate the plasmonic response of the cavity using only the dipolar plasmonic mode and calculate the linewidth maps in this single-mode approximation.

To describe the distribution of the electric field belonging to the dipolar plasmonic mode, we apply two successive
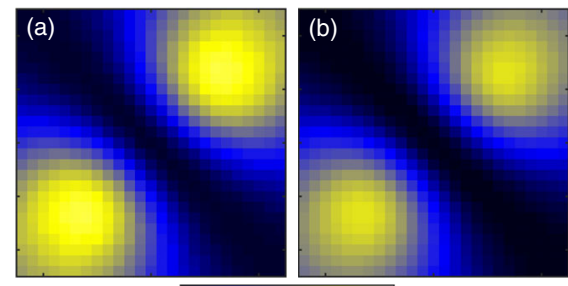

$0 \square 3 \mathrm{meV}$

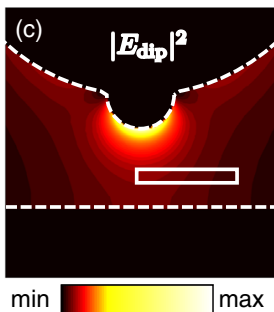

FIG. 12. Interpretation of linewidth maps. (a) Linewidth map calculated using the model described in Appendix A, and (b) linewidth map calculated using the single-mode approximation. (c) Field distribution $\left(\left|E_{\text {dip }}\right|^{2}\right)$ corresponding to the dipolar plasmon mode resonant with the molecular exciton. The size of the maps is $2.5 \times 2.5 \mathrm{~nm}^{2}$, and the size of panel (c) is $4 \times 4 \mathrm{~nm}^{2}$. computational steps. First, we resonantly excite the dipolar plasmon of the tip by applying a homogeneous electric field oriented along $z$ to the entire structure, including both the tip and the substrate. In the second step, we perform a separate simulation where we use the induced surface charge density of the dipolar plasmon of the tip obtained from the first step as a source of a quasistatic electric field assuming that the electric potential asymptotically decays to zero.

In order to obtain a quantitative comparison between the full theoretical model introduced in Appendix A and the single-mode approximation, we quantize the field distribution of the plasmonic dipolar mode. To that end, we calculate its effective mode volume, which, in the quasistatic limit (absence of magnetic fields), can be obtained by integrating the spatial distribution of the electric field $\mathbf{E}_{\text {calc }}$ obtained numerically [72]

$$
V_{\text {eff }}=\frac{\left.\iiint \frac{1}{2} \operatorname{Re}\left\{\frac{d[\omega \epsilon(\mathbf{r}, \omega)]}{d \omega}\right\}\right|_{\omega_{\mathrm{pl}}}\left|\mathbf{E}_{\mathrm{calc}}(\mathbf{r})\right|^{2} d^{3} \mathbf{r}}{\max \left(\left|\mathbf{E}_{\mathrm{calc}}(\mathbf{r})\right|^{2}\right)} .
$$

Here, $\varepsilon(\mathbf{r}, \omega)$ is the spatially dependent dielectric function of the environment, $\omega$ is the angular frequency, and the expression is evaluated for the resonance frequency of the dipolar plasmon $\omega=\omega_{\mathrm{pl}}$. For the dipolar plasmon of the picocavity tip and gap $=1.2 \mathrm{~nm}$, we obtain $V_{\text {eff }} \approx 21.5 \mathrm{~nm}^{3}$. The positive-frequency part of the quantized plasmonic potential of the mode can then be obtained as [72]

$$
\phi_{\text {dip }}(\mathbf{r})=\sqrt{\frac{\hbar \omega_{\mathrm{pl}}}{2 \varepsilon_{0} V_{\text {eff }}}} \frac{\phi_{\text {calc }}(\mathbf{r})}{\max \left\{\left|\mathbf{E}_{\text {calc }}(\mathbf{r})\right|\right\}},
$$

where $\mathbf{E}_{\text {calc }}=-\nabla \phi_{\text {calc }}$. Finally, the plasmon-induced linewidth can be calculated as

$$
\gamma_{\mathrm{eg}}(\mathbf{R}) \approx \frac{4|g(\mathbf{R})|^{2}}{\gamma_{\mathrm{pl}}}
$$

with

$$
\hbar g(\mathbf{R})=\iiint \rho_{\mathrm{eg}}(\mathbf{r}-\mathbf{R}) \phi_{\mathrm{dip}}(\mathbf{r}) d^{3} \mathbf{r}
$$

In Eqs. (F3) and (F4) we have again made explicit the dependence on the relative position $\mathbf{R}$ of the molecule with respect to the tip. We plot the plasmon-induced linewidth as calculated using the single-mode approximation in Fig. 12(b) and the corresponding field profile of the dipolar plasmon mode $\left(\left|\mathbf{E}_{\text {dip }}\right|^{2}=\left|\nabla \phi_{\text {dip }}\right|^{2}\right)$ in Fig. 12(c). In the calculation, we use $\hbar \gamma_{\mathrm{pl}}=34 \mathrm{meV}$ extracted from the data shown in Fig. 7. We note that the agreement between the linewidth maps calculated using both models proves the dominant role of the dipolar plasmonic mode in the formation of the STML linewidth maps. We also note that the quantized field 


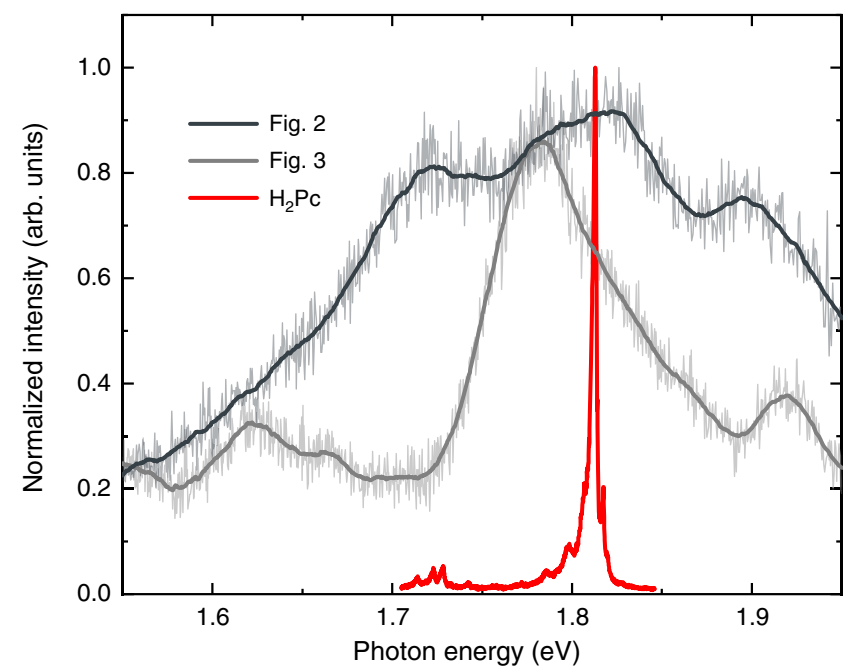

FIG. 13. Plasmonic spectra of the tips used to perform measurements shown in Figs. 2 and 3. The spectrum of the molecule is shown as a reference. Parameters for all spectra are as follows: $V=-2.5 \mathrm{~V}, I=100 \mathrm{pA}$.

distribution of the dipolar plasmon is an intrinsic property of the probe, i.e., of the tip and the substrate, and is independent of the position of the molecule. Broadly speaking, this can be understood in analogy to the standard interpretation of STM images, where it is the spatial distribution of the orbital of the tip that is responsible for the formation of STM maps.

\section{APPENDIX G: EXPERIMENTAL PLASMONIC RESONANCE}

In Fig. 13, we provide the reference plasmonic spectra of the tips used to record measurements presented in Figs. 2 and 3, compared with the spectrum recorded on top of the $\mathrm{H}_{2} \mathrm{Pc}$ molecule. The plasmonic resonance in our experiments is at the energy of the molecular exciton or slightly shifted, thus justifying the parameters used for the calculations that assume a tip with resonance energy matching the molecular exciton (see Appendix A).

[1] W. E. Moerner and L. Kador, Optical Detection and Spectroscopy of Single Molecules in a Solid, Phys. Rev. Lett. 62, 2535 (1989).

[2] M. Orrit and J. Bernard, Single Pentacene Molecules Detected by Fluorescence Excitation in a p-Terphenyl Crystal, Phys. Rev. Lett. 65, 2716 (1990).

[3] B. Huang, M. Bates, and X. Zhuang, Super-Resolution Fluorescence Microscopy, Annu. Rev. Biochem. 78, 993 (2009).

[4] S. W. Hell et al., The 2015 Super-Resolution Microscopy Roadmap, J. Phys. D 48, 443001 (2015).

[5] B. Lounis and W. E. Moerner, Single Photons on Demand from a Single Molecule at Room Temperature, Nature (London) 407, 491 (2000).
[6] C. Toninelli, I. Gerhardt, A. S. Clark, A. Reserbat-Plantey, S. Götzinger, Z. Ristanović, M. Colautti, P. Lombardi, K. Major, I. Deperasińska, W. H. Pernice, F. H. L. Koppens, B. Kozankiewicz, A. Gourdon, V. Sandoghdar, and M. Orrit, Single Organic Molecules for Photonic Quantum Technologies, Nat. Mater. 20, 1615 (2021).

[7] E. M. Purcell, H. C. Torrey, and R. V. Pound, Resonance Absorption by Nuclear Magnetic Moments in a Solid, Phys. Rev. 69, 37 (1946).

[8] L. Novotny and B. Hecht, Principles of Nano-Optics (Cambridge University Press, Cambridge, England, 2006).

[9] W. E. Lamb and R. C. Retherford, Fine Structure of the Hydrogen Atom by a Microwave Method, Phys. Rev. 72, 241 (1947).

[10] H. A. Bethe, The Electromagnetic Shift of Energy Levels, Phys. Rev. 72, 339 (1947).

[11] H. A. Bethe, L. M. Brown, and J. R. Stehn, Numerical Value of the Lamb Shift, Phys. Rev. 77, 370 (1950).

[12] E. A. Power, Zero-Point Energy and the Lamb Shift, Am. J. Phys. 34, 516 (1966).

[13] J. Stark, Beobachtungen über den Effekt des Elektrischen Feldes auf Spektrallinien. I. Quereffekt, Ann. Phys. (Berlin) 348, 965 (1914).

[14] R. V. Krems, DC Stark Effect, in Molecules in Electromagnetic Fields (John Wiley \& Sons, New York, 2018), Chap. 2, pp. 35-58.

[15] L. Limot, T. Maroutian, P. Johansson, and R. Berndt, Surface-State Stark Shift in a Scanning Tunneling Microscope, Phys. Rev. Lett. 91, 196801 (2003).

[16] J. Martínez-Blanco, C. Nacci, S. C. Erwin, K. Kanisawa, E. Locane, M. Thomas, F. von Oppen, P. W. Brouwer, and S. Fölsch, Gating a Single-Molecule Transistor with Individual Atoms, Nat. Phys. 11, 640 (2015).

[17] I. Fernández-Torrente, D. Kreikemeyer-Lorenzo, A. Stróżecka, K. J. Franke, and J. I. Pascual, Gating the Charge State of Single Molecules by Local Electric Fields, Phys. Rev. Lett. 108, 036801 (2012).

[18] E. Betzig and R. J. Chichester, Single Molecules Observed by Near-Field Scanning Optical Microscopy, Science 262, 1422 (1993).

[19] F. Keilmann and R. Hillenbrand, Near-Field Microscopy by Elastic Light Scattering from a Tip, Phil. Trans. R. Soc. A 362, 787 (2004).

[20] H. G. Frey, S. Witt, K. Felderer, and R. Guckenberger, HighResolution Imaging of Single Fluorescent Molecules with the Optical Near-Field of a Metal Tip, Phys. Rev. Lett. 93, 200801 (2004).

[21] L. Novotny and S. J. Stranick, Near-Field Optical Microscopy and Spectroscopy with Pointed Probes, Annu. Rev. Phys. Chem. 57, 303 (2006).

[22] S. Kühn, U. Håkanson, L. Rogobete, and V. Sandoghdar, Enhancement of Single-Molecule Fluorescence Using a Gold Nanoparticle as an Optical Nanoantenna, Phys. Rev. Lett. 97, 017402 (2006).

[23] A. Hartschuh, Tip-Enhanced Near-Field Optical Microscopy, Angew. Chem., Int. Ed. Engl. 47, 8178 (2008).

[24] N. Mauser and A. Hartschuh, Tip-Enhanced NearField Optical Microscopy, Chem. Soc. Rev. 43, 1248 (2014).

[25] K.-D. Park, T. Jiang, G. Clark, X. Xu, and M. B. Raschke, Radiative Control of Dark Excitons at Room Temperature 
by Nano-Optical Antenna-Tip Purcell Effect, Nat. Nanotechnol. 13, 59 (2018).

[26] R. Zhang, Y. Zhang, Z. C. Dong, S. Jiang, C. Zhang, L. G. Chen, L. Zhang, Y. Liao, J. Aizpurua, Y. Luo, J. L. Yang, and J. G. Hou, Chemical Mapping of a Single Molecule by Plasmon-Enhanced Raman Scattering, Nature (London) 498, 82 (2013).

[27] J. Lee, K. T. Crampton, N. Tallarida, and V. A. Apkarian, Visualizing Vibrational Normal Modes of a Single Molecule with Atomically Confined Light, Nature (London) 568, 78 (2019).

[28] B. Yang, G. Chen, A. Ghafoor, Y. Zhang, Y. Zhang, Y. Zhang, Y. Luo, J. Yang, V. Sandoghdar, J. Aizpurua, Z. Dong, and J. G. Hou, Sub-Nanometre Resolution in SingleMolecule Photoluminescence Imaging, Nat. Photonics 14, 693 (2020).

[29] X. H. Qiu, G. V. Nazin, and W. Ho, Vibrationally resolved fluorescence excited with submolecular precision, Science 299, 542 (2003).

[30] C. Chen, P. Chu, C. A. Bobisch, D. L. Mills, and W. Ho, Viewing the Interior of a Single Molecule: Vibronically Resolved Photon Imaging at Submolecular Resolution, Phys. Rev. Lett. 105, 217402 (2010).

[31] M. C. Chong, G. Reecht, H. Bulou, A. Boeglin, F. Scheurer, F. Mathevet, and G. Schull, Narrow-Line Single-Molecule Transducer between Electronic Circuits and Surface Plasmons, Phys. Rev. Lett. 116, 036802 (2016).

[32] M. C. Chong, L. Sosa-Vargas, H. Bulou, A. Boeglin, F. Scheurer, F. Mathevet, and G. Schull, Ordinary and Hot Electroluminescence from Single-Molecule Devices: Controlling the Emission Color by Chemical Engineering, Nano Lett. 16, 6480 (2016).

[33] Y. Zhang, Y. Luo, Y. Zhang, Y.-J. Yu, Y.-M. Kuang, L. Zhang, Q.-S. Meng, Y. Luo, J.-L. Yang, Z.-C. Dong, and J. G. Hou, Visualizing Coherent Intermolecular DipoleDipole Coupling in Real Space, Nature (London) 531, 623 (2016).

[34] H. Imada, K. Miwa, M. Imai-Imada, S. Kawahara, K. Kimura, and Y. Kim, Real-Space Investigation of Energy Transfer in Heterogeneous Molecular Dimers, Nature (London) 538, 364 (2016).

[35] C. Große, P. Merino, A. Rosławska, O. Gunnarsson, K. Kuhnke, and K. Kern, Submolecular Electroluminescence Mapping of Organic Semiconductors, ACS Nano 11, 1230 (2017).

[36] B. Doppagne, M. C. Chong, E. Lorchat, S. Berciaud, M. Romeo, H. Bulou, A. Boeglin, F. Scheurer, and G. Schull, Vibronic Spectroscopy with Submolecular Resolution from STM-Induced Electroluminescence, Phys. Rev. Lett. 118, 127401 (2017).

[37] H. Imada, K. Miwa, M. Imai-Imada, S. Kawahara, K. Kimura, and Y. Kim, Single-Molecule Investigation of Energy Dynamics in a Coupled Plasmon-Exciton System, Phys. Rev. Lett. 119, 013901 (2017).

[38] Y. Zhang, Q.-S. Meng, L. Zhang, Y. Luo, Y.-J. Yu, B. Yang, Y. Zhang, R. Esteban, J. Aizpurua, Y. Luo, J.-L. Yang, Z.-C. Dong, and J.G. Hou, Sub-Nanometre Control of the Coherent Interaction between a Single Molecule and a Plasmonic Nanocavity, Nat. Commun. 8, 15225 (2017).
[39] L. Zhang, Y.-J. Yu, L.-G. Chen, Y. Luo, B. Yang, F.-F. Kong, G. Chen, Y. Zhang, Q. Zhang, Y. Luo, J.-L. Yang, Z.-C. Dong, and J. G. Hou, Electrically Driven Single-Photon Emission from an Isolated Single Molecule, Nat. Commun. 8, 580 (2017).

[40] B. Doppagne, M. C. Chong, H. Bulou, A. Boeglin, F. Scheurer, and G. Schull, Electrofluorochromism at the Single-Molecule Level, Science 361, 251 (2018).

[41] J. Doležal, P. Merino, J. Redondo, L. Ondič, A. Cahlík, and M. Švec, Charge Carrier Injection Electroluminescence with CO-Functionalized Tips on Single Molecular Emitters, Nano Lett. 19, 8605 (2019).

[42] B. Doppagne, T. Neuman, R. Soria-Martinez, L. E. P. López, H. Bulou, M. Romeo, S. Berciaud, F. Scheurer, J. Aizpurua, and G. Schull, Single-Molecule Tautomerization Tracking through Space- and Time-Resolved Fluorescence Spectroscopy, Nat. Nanotechnol. 15, 207 (2020).

[43] V. Rai, L. Gerhard, Q. Sun, C. Holzer, T. Repän, M. Krstić, L. Yang, M. Wegener, C. Rockstuhl, and W. Wulfhekel, Boosting Light Emission from Single Hydrogen Phthalocyanine Molecules by Charging, Nano Lett. 20, 7600 (2020).

[44] J. Doležal, S. Canola, P. Merino, and M. Švec, ExcitonTrion Conversion Dynamics in a Single Molecule, ACS Nano 15, 7694 (2021).

[45] S. Cao, A. Rosławska, B. Doppagne, M. Romeo, M. Féron, F. Chérioux, H. Bulou, F. Scheurer, and G. Schull, Energy Funneling within Multi-Chromophore Architectures Monitored with Sub-Nanometer Resolution, Nat. Chem. 13, 766 (2021).

[46] T.-C. Hung, B. Kiraly, J. H. Strik, A. A. Khajetoorians, and D. Wegner, Plasmon-Driven Motion of an Individual Molecule, Nano Lett. 21, 5006 (2021).

[47] K. Kuhnke, V. Turkowski, A. Kabakchiev, T. Lutz, T. S. Rahman, and K. Kern, Pentacene Excitons in Strong Electric Fields, Chem. Phys. Chem. 19, 277 (2018).

[48] H. Imada, M. Imai-Imada, K. Miwa, H. Yamane, T. Iwasa, Y. Tanaka, N. Toriumi, K. Kimura, N. Yokoshi, A. Muranaka, M. Uchiyama, T. Taketsugu, Y. K. Kato, H. Ishihara, and Y. Kim, Single-Molecule Laser Nanospectroscopy with Micro-Electron Volt Energy Resolution, Science 373, 95 (2021).

[49] F. Benz, M. K. Schmidt, A. Dreismann, R. Chikkaraddy, Y. Zhang, A. Demetriadou, C. Carnegie, H. Ohadi, B. de Nijs, R. Esteban, J. Aizpurua, and J. J. Baumberg, SingleMolecule Optomechanics in "Picocavities," Science 354, 726 (2016).

[50] M. Barbry, P. Koval, F. Marchesin, R. Esteban, A. G. Borisov, J. Aizpurua, and D. Sánchez-Portal, Atomistic Near-Field Nanoplasmonics: Reaching Atomic-Scale Resolution in Nanooptics, Nano Lett. 15, 3410 (2015).

[51] M. Urbieta, M. Barbry, Y. Zhang, P. Koval, D. SánchezPortal, N. Zabala, and J. Aizpurua, Atomic-Scale Lightning Rod Effect in Plasmonic Picocavities: A Classical View to a Quantum Effect, ACS Nano 12, 585 (2018).

[52] T. P. Rossi, T. Shegai, P. Erhart, and T. J. Antosiewicz, Strong Plasmon-Molecule Coupling at the Nanoscale Revealed by First-Principles Modeling, Nat. Commun. 10, 1 (2019). 
[53] T. Wu, W. Yan, and P. Lalanne, Bright Plasmons with Cubic Nanometer Mode Volumes through Mode Hybridization, ACS Photonics 8, 307 (2021).

[54] A. Rosławska, P. Merino, A. Grewal, C. C. Leon, K. Kuhnke, and K. Kern, Atomic-Scale Structural Fluctuations of a Plasmonic Cavity, Nano Lett. 21, 7221 (2021).

[55] J. J. Baumberg, J. Aizpurua, M. H. Mikkelsen, and D. R. Smith, Extreme Nanophotonics from Ultrathin Metallic Gaps, Nat. Mater. 18, 668 (2019).

[56] C. Carnegie, J. Griffiths, B. de Nijs, C. Readman, R. Chikkaraddy, W. M. Deacon, Y. Zhang, I. Szab, E. Rosta, J. Aizpurua, and J. J. Baumberg, Room-Temperature Optical Picocavities below $1 \mathrm{~nm}^{3}$ Accessing Single-Atom Geometries, J. Phys. Chem. Lett. 9, 7146 (2018).

[57] M. Valiev, E. J. Bylaska, N. Govind, K. Kowalski, T. P. Straatsma, H. J. Van Dam, D. Wang, J. Nieplocha, E. Apra, T. L. Windus, and W. A. de Jong, NWChem: A Comprehensive and Scalable Open-Source Solution for Large Scale Molecular Simulations, Comput. Phys. Commun. 181, 1477 (2010).

[58] M. Sibata, A. Tedesco, and J. Marchetti, Photophysicals and Photochemicals Studies of Zinc(II) Phthalocyanine in Long Time Circulation Micelles for Photodynamic Therapy Use, European Journal of pharmaceutical sciences 23, 131 (2004).

[59] B. W. Caplins, T. K. Mullenbach, R. J. Holmes, and D. A. Blank, Femtosecond to Nanosecond Excited State Dynamics of Vapor Deposited Copper Phthalocyanine Thin Films, Phys. Chem. Chem. Phys. 18, 11454 (2016).

[60] A. Delga, J. Feist, J. Bravo-Abad, and F. J. Garcia-Vidal, Theory of Strong Coupling between Quantum Emitters and Localized Surface Plasmons, J. Opt. 16, 114018 (2014).

[61] T. Neuman, R. Esteban, D. Casanova, F. J. García-Vidal, and J. Aizpurua, Coupling of Molecular Emitters and Plasmonic Cavities beyond the Point-Dipole Approximation, Nano Lett. 18, 2358 (2018).

[62] J. Tersoff and D. R. Hamann, Theory of the Scanning Tunneling Microscope, Phys. Rev. B 31, 805 (1985).
[63] C. Dutreix, R. Avriller, B. Lounis, and F. Pistolesi, Two-Level System as Topological Actuator for Nanomechanical Modes, Phys. Rev. Research 2, 023268 (2020).

[64] S. Das, V. E. Elfving, S. Faez, and A. S. Sørensen, Interfacing Superconducting Qubits and Single Optical Photons Using Molecules in Waveguides, Phys. Rev. Lett. 118, 140501 (2017).

[65] A. Laucht, F. Hofbauer, N. Hauke, J. Angele, S. Stobbe, M. Kaniber, G. Böhm, P. Lodahl, M.-C. Amann, and J. J. Finley, Electrical Control of Spontaneous Emission and Strong Coupling for a Single Quantum Dot, New J. Phys. 11, 023034 (2009).

[66] H.-P. Breuer and F. Petruccione, The Theory of Open Quantum Systems (Oxford University Press, New York, 2003).

[67] A. Rosławska, P. Merino, C. C. Leon, A. Grewal, M. Etzkorn, K. Kuhnke, and K. Kern, Gigahertz Frame Rate Imaging of Charge-Injection Dynamics in a Molecular Light Source, Nano Lett. 21, 4577 (2021).

[68] F. Aguilar-Galindo, A. G. Borisov, and S. Daz-Tendero, Unveiling the Anisotropic Behavior of Ultrafast Electron Transfer at the Metal/Organic Interface, Appl. Surf. Sci. 554, 149311 (2021).

[69] P. B. Johnson and R. W. Christy, Optical Constants of the Noble Metals, Phys. Rev. B 6, 4370 (1972).

[70] C. Murray, N. Dozova, J. G. McCaffrey, N. Shafizadeh, W. Chin, M. Broquier, and C. Crepin, Visible Luminescence Spectroscopy of Free-Base and Zinc Phthalocyanines Isolated in Cryogenic Matrices, Phys. Chem. Chem. Phys. 13, 17543 (2011).

[71] G. Raunio, L. Almqvist, and R. Stedman, Phonon Dispersion Relations in $\mathrm{NaCl}$, Phys. Rev. 178, 1496 (1969).

[72] R. Esteban, J. Aizpurua, and G. W. Bryant, Strong Coupling of Single Emitters Interacting with Phononic Infrared Antennae, New J. Phys. 16, 013052 (2014). 